\title{
Acetalated Dextran Microparticles for Codelivery of STING and TLR7/8 Agonists
}

\author{
Michael A. Collier, ${ }^{\dagger, \nabla}$ Robert D. Junkins, ${ }^{\ddagger, \nabla}$ Matthew D. Gallovic, ${ }^{\dagger}$ Brandon M. Johnson, ${ }^{\ddagger}$ \\ Monica M. Johnson, ${ }^{\dagger}$ Andrew N. Macintyre, ${ }^{\S}$ Gregory D. Sempowski, ${ }^{\S}$ Eric M. Bachelder, ${ }^{\dagger}$ \\ Jenny P.-Y. Ting, \\ ${ }^{\dagger}$ Eshelman School of Pharmacy, Division of Pharmacoengineering and Molecular Pharmaceutics, ${ }^{\ddagger}$ Department of Genetics, \\ Lineberger Comprehensive Cancer Center, "Department of Microbiology and Immunology, ${ }^{\perp}$ Institute for Inflammatory Diseases, \\ and ${ }^{\#}$ Center for Translational Immunology, University of North Carolina, Chapel Hill, North Carolina 27599, United States \\ ${ }^{\S}$ Duke Human Vaccine Institute, Duke University Medical Center, Durham, North Carolina 27710, United States
}

\begin{abstract}
Vaccines are the most effective tool for preventing infectious diseases; however, subunit vaccines, considered the safest type, suffer from poor immunogenicity and require adjuvants to create a strong and sustained immune response. As adjuvants, pathogen-associated molecular patterns (PAMPs) offer potent immunostimulatory properties and defined mechanisms of action through their cognate pattern recognition receptors (PRRs). Their activity

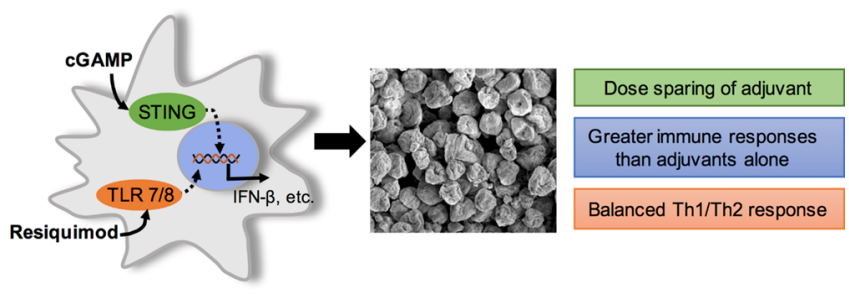
can be further enhanced through combining two or more PAMPs, particularly those that activate multiple immune signaling pathways. However, the cytosolic localization of many PRRs requires intracellular delivery of PAMPs for optimal biological activity, which is particularly true of the stimulator of interferon genes (STING) PRR. Using acetalated dextran (Ace-DEX) microparticles (MPs) encapsulating STING agonist 3'3'-cyclic GMP-AMP (cGAMP) combined with soluble PAMPS, we screened the effect of codelivery of adjuvants using primary mouse bone marrow derived dendritic cells (BMDCs). We identified that codelivery of cGAMP MPs and soluble Toll-like receptor $7 / 8$ (TLR7/8) agonist resiquimod (R848) elicited the broadest cytokine response. cGAMP and R848 were then coencapsulated within Ace-DEX MPs via electrospray. Using the model antigen ovalbumin, we observed that Ace-DEX MPs coencapsulating cGAMP and R848 (cGAMP/R848 Ace-DEX MPs) induced antigen-specific cellular immunity, and a balanced Th1/Th2 humoral response that was greater than cGAMP Ace-DEX MPs alone and PAMPs delivered in separate MPs. These data indicate that polymeric Ace-DEX MPs loaded with STING and TLR7/8 agonists represent a potent cellular and humoral vaccine adjuvant.
\end{abstract}

KEYWORDS: acetalated dextran, vaccine adjuvants, microparticles, STING, cGAMP adjuvant

\section{INTRODUCTION}

Vaccination is widely regarded as the most efficient strategy for preventing morbidity and mortality associated with infectious disease. Historically, protection has been achieved using live attenuated vaccines (LAV) or inactivated pathogens, which can induce long-lasting and potent immunity. However, LAVs have safety concerns such as risk of reversion to a more virulent strain, which limits their ability to be broadly applied to populations such as the youth, elderly, and immunocompromised. ${ }^{1,2}$ Inactivated pathogen vaccines overcome some of the safety concerns associated with LAVs; however, they are less immunogenic, and like LAVs, they fail to provide protection against pathogens with high levels of antigenic variability. A safer alternative to LAVs or inactivated pathogen vaccines are subunit vaccines, which contain only part of the pathogen. Subunit antigens tend to be poorly immunogenic, requiring the administration of an adjuvant to generate protective immunity. ${ }^{3}$ While existing FDA-approved adjuvants such as aluminum salts (alum) or MF59 can generate humoral immunity and $\mathrm{T}$ helper cell type 2 (Th2)-skewed immune responses, they frequently fail to induce significant $\mathrm{T}$ helper cell type 1 (Th1)-biased immunity. A Th1 response is crucial for protective cellular immunity against intracellular pathogens, like viruses. ${ }^{4}$ Furthermore, the mechanisms of alum and MF59 are poorly understood. ${ }^{5}$ Therefore, vaccine adjuvants with known mechanisms that mount strong Th1 humoral and cellular responses are needed.

Pathogen-associated molecular patterns (PAMPs) have garnered a great deal of interest as vaccine adjuvants, because they have known mechanisms and can invoke a Th1 immune response. PAMPs consist of one or more structures that are

Received: June 4, 2018

Revised: September 27, 2018

Accepted: October 3, 2018

Published: October 3, 2018 
sensed by pattern recognition receptors (PRRs) on host cells and can activate immune responses mediated through one or more signaling pathways. Three common classes of PRRs are Toll-like receptors (TLRs), nucleotide-binding oligomerization domain (NOD)-like receptors (NLRs), and stimulator of interferon genes (STING). Some PRRs are found on the plasma membrane (e.g., TLR2, TLR4), where they are readily accessible to extracellularly delivered PAMPs, such as MPL, an FDA-approved agonist for TLR4. In contrast, others PRRs are located on phagasomal membranes or in the cytosol and require intracellular delivery of PAMPs such as R848, murabutide, and poly I:C, which activate TLR7/8, NOD2, and TLR3, respectively. These cytosolic PRRs predominantly coordinate Th1-biased humoral and cellular immunity. ${ }^{6}$ Of these cytosolic PRRs, STING activation can result in potent Th1-biased immune responses. One ligand that binds directly to and activates STING is $3^{\prime}, 3^{\prime}$-cyclic GMP-AMP (cGAMP). This variety of cGAMP, which is a cyclic dinucleotide (CDN) made by bacteria, has a higher STING binding affinity than other bacterial $\mathrm{CDNs}^{7}$ and has been shown to generate robust transcriptional activation of the type-I interferon (IFN) pathway. ${ }^{8}$ While cGAMP is known to be a potent STING agonist, the cellular membrane is a formidable obstacle to overcome in order for this hydrophilic charged small molecule to bind to its cytosolic PRR.

To improve delivery of cytosolic PAMPs such as GAMP and other STING agonist cyclic dinucleotides (CDNs), carriers such as liposomes ${ }^{9-12}$ and polymeric particles ${ }^{13,14}$ have been applied in preclinical studies. However, liposomes are difficult to scale for vaccine applications, ${ }^{15}$ and the polymers employed to date require polymer blends (e.g., polyethylenimine/ hyaluronic acid) ${ }^{12}$ or are known to be cytotoxic (e.g., poly beta-amino esters). ${ }^{13}$ Polyesters (e.g., poly lactic-co-glycolic acid [PLGA]) are an another attractive polymer type, but disadvantages such as slow degradation rates within phagosomal compartments of antigen-presenting cells (APCs) and acidic hydrolytic byproducts limit their clinical efficacy. ${ }^{16,17}$

To overcome the shortcomings of these delivery vehicles, we have utilized the biopolymer acetalated dextran (Ace-DEX), which is derived from the FDA-approved water-soluble polysaccharide, dextran. ${ }^{18-20}$ Ace-DEX is unique due to its simple synthesis, in which acetal groups are formed along the glucose backbone of dextran, making the polymer acidsensitive and organic-soluble. In aqueous environments, acetal groups are hydrolytically cleaved, generating $\mathrm{pH}$-neutral and biocompatible degradation byproducts ethanol, acetone, and dextran. Ace-DEX has been formulated into microparticles (MPs), which offer micron particle size-dependent passive delivery to phagocytic APCs, ${ }^{20,21}$ resulting in minimal offtarget delivery and efficient trafficking to lymph nodes. ${ }^{22,23}$ Ace-DEX MPs have demonstrated rapid degradation over a period of hours to days within low $\mathrm{pH}$ lysosomal conditions, with sustained degradation over a period of days to months at physiological $\mathrm{pH}^{18,24}$ Ace-DEX MPs have illustrated dose sparing of several PAMPs ${ }^{24-28}$ as well as subunit vaccines against Bacillus anthracis, Burkholderia pseudomallei, and influenza. ${ }^{27,29,30}$

Previously, through electro-hydrodynamic spraying (electrospray), we have generated cGAMP-loaded Ace-DEX MPs ranging in size from 1.5 to $3 \mu \mathrm{m} .^{22}$ A comparative study evaluating cGAMP encapsulated in liposomes, PLGA MPs, and Ace-DEX MPs demonstrated a significantly higher expression of type-I IFN in response to Ace-DEX MPs than the other formulations. Additionally, cGAMP Ace-DEX MPs generated a robust cellular response and a balanced Th1/Th2 humoral immune response, which provided long-term (7 months) protection against a lethal influenza challenge. However, despite this success, we noted that the breadth of cytokines elicited by cGAMP Ace-DEX MPs was relatively narrow and did not include cytokines known to contribute to robust and long-lasting immune responses, most notably IL- $1 \beta$ and IL-12..$^{31-34}$

One approach for eliciting broader and more protective cytokine responses is codelivery of PAMPS for concurrent activation of PRRs. During natural infection, pathogens often stimulate multiple PRRs, ${ }^{35}$ illustrating the importance of vaccines that can mimic this response to provide protection. An example of a multi-PRR stimulating vaccine is the yellow fever 17D (YF-17D) vaccine. The FDA-approved LAV YF$17 \mathrm{D}$ vaccine is one of the most effective vaccines known to date and activates multiple TLRs to generate a potent and balanced Th1/Th2 response. ${ }^{36}$ The broadly stimulating capacity of YF-17D vaccine yields a potent vaccine that only needs to be administered once for life-long protection. This is because cross-talk between multiple PRRs can be both quantitatively and qualitatively different than the contribution of each individual pathway. ${ }^{37}$

This current study first investigated the formulation of cGAMP MPs using multiple techniques and evaluated in vitro combinations of soluble PAMP adjuvants for synergistic potential. The optimal ratio of this multiadjuvant system was determined and then formulated into a single MP delivery vehicle containing two PAMPs. An in vitro comparison of a single adjuvant and combinational adjuvants using Ace-DEX and PLGA MPs was performed. The adjuvant systems also were compared in vivo to determine the degree of immune response enhancement.

\section{MATERIALS AND METHODS}

Animal Ethics Statement. All studies were conducted in accordance with National Institutes of Health guidelines for the care and use of laboratory animals and approved by the Institutional Animal Care and Use Committee at the University of North Carolina. All animals were maintained in pathogen-free facilities.

Chemicals. All materials were purchased from SigmaAldrich (St. Louis, MO) and used as received, unless otherwise indicated. Vaccine grade ovalbumin (OVA), Alhydrogel 2\%, cGAMP, and resiquimod (R848) were purchased from Invivogen (San Diego, CA).

Synthesis of Acetalated Dextran. Ace-DEX was synthesized according to Kauffman et al. using dextran from Leuconostoc mesenteroides (molecular weight $=70 \mathrm{kDa}) .^{38}$ Briefly, lyophilized dextran and pyridinium $p$-toluenesulfonate $(0.0617 \mathrm{mmol})$ were dissolved in anhydrous dimethyl sulfoxide (DMSO). Dissolved dextran was reacted with 2-ethoxypropene (Matrix, Columbia, SC) under nitrogen gas at room temperature for $30 \mathrm{~min}$ and quenched with an excess of triethylamine (TEA). The reaction volumes were then precipitated in basic water $(0.04 \%$ TEA by volume in water) and centrifuged, and the resulting pellet was frozen and lyophilized overnight. The following day, the product was dissolved in ethanol and centrifuged to further purify the polymer. The supernatant was precipitated in basic water, centrifuged again, frozen, and lyophilized to yield $70 \mathrm{kDa}$ Ace- 
DEX polymer. The polymer's relative cyclic acetal coverage was determined to be $40 \%$, as measured by ${ }^{1} \mathrm{H}$ NMR spectroscopy (Inova $400 \mathrm{MHz}$ spectrometer) following degradation of the polymer in $10 \% \mathrm{v} / \mathrm{v}$ deuterium chloride in deuterium oxide.

Formulation of Microparticles Using Ace-DEX or PLGA by Electrospray or Emulsion. To prevent endotoxin contamination, all dishes and glassware were soaked in $1.0 \mathrm{M}$ sodium hydroxide overnight, washed with isopropanol, and dried before use. Encapsulation of cGAMP or R848 within Ace-DEX microparticles (cGAMP Ace-DEX MPs or R848 Ace-DEX MPs, respectively) or cGAMP and R848 coencapsulated within the same MPs (cGAMP/R848 Ace-DEX MPs) were fabricated using electrospray following the setup of Junkins et al. $^{22}$ The same methods were used to make poly(lactic-co-glycolic acid) ([PLGA], 85:15, 50-75 kDa, ester terminated) particles coencapsulating cGAMP and R848 (cGAMP/R848 PLGA MPs). ${ }^{22}$ Egg phosphatidylcholine (EggPC, Avanti Polar Lipids, Alabaster, AL) was added postfabrication to increase MP suspendability.

Alternatively, fabrication of MPs by solvent evaporation was performed using a modified version of the water-in-oil-in-water emulsion procedure previously reported. ${ }^{39}$

The endotoxin content of all MPs was measured prior to treatment according to Gallovic et al. ${ }^{39}$ and contained $<0.25$ $\mathrm{EU} / \mathrm{mg}$, within the recommended levels for preclinical subunit vaccine formulations. ${ }^{40}$

Imaging of Microparticles. cGAMP, R848, and combination cGAMP/R848 MPs were imaged using a Hitachi S4700 Cold Cathode Field Emission Scanning Electron Microscope (SEM). SEM images were used to confirm MP size and morphology.

Quantification of cGAMP and R848 Loading within Microparticles. cGAMP loading was determined using high performance liquid chromatography (HPLC) according to Junkins et al. ${ }^{22}$ R848 loading was determined by its autofluorescence (ex/em: 260/360 nm) according to Duong et al. ${ }^{28}$ Blank MPs were used as a background correction.

cGAMP and R848 Release Profiles from Microparticles. cGAMP, R848, or cGAMP/R848 Ace-DEX MPs were suspended in phosphate-buffered saline (PBS; $\mathrm{pH} 7.4$ ) at $37{ }^{\circ} \mathrm{C}$ on a shaker plate operating at $200 \mathrm{rpm}$. At predetermined time points, aliquots were removed and centrifuged $\left(30 \mathrm{~min}\right.$ at $21000 \mathrm{~g}, 4{ }^{\circ} \mathrm{C}$ ). The pellets were analyzed similarly to the loading protocols outlined above for either cGAMP or R848 MPs, respectively. Blank MPs collected at each time point were subjected to the same process and used as background subtractions.

Flow Cytometry of Multiple Fluorophore Microparticles. Ace-DEX encapsulating fluorophores BODIPY 493/503, Texas Red, and BODIPY 630/650 (Thermo Fisher Scientific) were coencapsulated within the same MPs using the electrospray setup as described above. MPs were suspended in FACs buffer (PBS containing 4\% fetal calf serum and $0.05 \%$ sodium azide), and flow cytometric analysis was performed using an LSR II flow cytometer (Becton Dickinson, Franklin Lakes, NJ). Blank MPs were used to generate a gated population.

Bone Marrow Derived Dendritic Cell Culture. Bone marrow derived dendritic cells (BMDCs) were cultured as described previously. ${ }^{41}$ Briefly, bone marrow obtained from 8to 12 -week-old C57BL/6 mice were washed and seeded in BMDC differentiation media containing mouse GM-CSF
(Peprotech, Rocky Hill, NJ). After 7 days, nonadherent cells were seeded in GM-CSF-free media overnight prior to treatment.

Cytokine ELISAs. Tumor necrosis factor (TNF), IL-6, IL$1 \beta$, IL-2, and IL-12p70 levels were measured by ELISAs purchased from BD Biosciences (San Jose, CA; Santa-Cruz, Dallas, TX), while interferon gamma (IFN- $\gamma$ ) was measured using ELISAs purchased from eBioscience (Thermo Fisher, Waltham, MA). ELISAs were performed according to the manufacturer's protocol. IFN- $\beta$ was measured via an ELISA described previously. ${ }^{22}$

In Vitro Assessment of Microparticle Activity. For in vitro screening of PAMPs with cGAMP MPs, BMDCs were treated with soluble agonists: murabutide $(10 \mu \mathrm{g} / \mathrm{mL})$, monophosphoryl lipid A (MPL, $1 \mu \mathrm{g} / \mathrm{mL})$, poly(dA:dT) $(10$ $\mu \mathrm{g} / \mathrm{mL}), \mathrm{CpG}(1 \mu \mathrm{g} / \mathrm{mL})$, poly(I:C) $(10 \mu \mathrm{g} / \mathrm{mL})$, or $\mathrm{R} 848$ $(0.01 \mu \mathrm{g} / \mathrm{mL})$ alone or in combination with cGAMP MPs (1 $\mu \mathrm{g} / \mathrm{mL}$ cGAMP) or an equivalent amount of blank MPs. Cell supernatants were collected $22 \mathrm{~h}$ later for cytokine analysis. Experiments with PLGA MPs and Ace-DEX MPs for comparison of coloaded and individually loaded particles were performed as with the adjuvant combinations. All experiments were performed in triplicate on four separate cultures of BMDCs.

In Vivo Immunization Studies. Age (8-16 weeks) and sex matched (male and female) C57BL/6 mice were obtained from Jackson Laboratories (Bar Harbor, ME). Mice were immunized by intramuscular (i.m.) injections on days 0 and 21 with either PBS or $10 \mu \mathrm{g}$ of soluble OVA (low endotoxin) mixed with an MP group (Supplemental Table S1) in a total volume of $50 \mu \mathrm{L}(n=4-5 /$ group $)$. Alum controls received soluble OVA plus Alhydrogel 2\% mixed at a 1:1 ratio by volume, also given as a $50 \mu \mathrm{L}$ i.m. injection. Serum was collected on day 28 for antibody titers. Mice received a second vaccine boost on day 35 and were then sacrificed on day 42 .

Antigen-Specific Serum Endpoint-Binding Titers. Antigen-specific serum antibody-binding titers (endpoint) were determined by a standard ELISA as previously described. ${ }^{42}$ Briefly, serial dilutions of test sera were performed in plates coated with OVA (Invivogen) at $2.5 \mu \mathrm{g} / \mathrm{mL}$. Following incubation and washing, horseradish-peroxidaseconjugated antimouse Ig-specific antibodies (Southern Biotech, Birmingham, AL) were added to plates at a 1:4000 dilution. After incubation and washing, 3,3',5,5'-tetramethylbenzidine substrate solution (KPL, Gaithersburg, MD) was added and stopped with $2 \mathrm{~N} \mathrm{H}_{2} \mathrm{SO}_{4}$ solution. The plates were read at an optical density (OD) of $450 \mathrm{~nm}$ (SynergyH1 plate reader, BioTek, Winooski, VT). Endpoint titers are reported as the log of the reciprocal of the highest serum dilution, at which the $O D$ value was equal to or greater than 3 times the average background OD of the plate.

Splenocyte Restimulation. On day 42, splenocytes were isolated from mice that were immunized as described above per Junkins et al. ${ }^{22}$ IFN- $\gamma$ and IL-2 ELISpots were performed according to manufacturer's protocols (Thermo Fisher Scientific) using $2 \times 10^{5}$ splenocytes stimulated with $10 \mu \mathrm{g} /$ $\mathrm{mL}$ SIINFEKL peptide (Anaspec, Fremont, CA) for $36 \mathrm{~h}$. Plates were dried, and spots were quantified using an ELISpot Reader System (AID, Strassberg, Germany). Alternatively, $2 \times$ $10^{5}$ splenocytes were stimulated with $10 \mu \mathrm{g} / \mathrm{mL}$ whole OVA protein for $36 \mathrm{~h}$. Supernatants were collected and analyzed for IL- 2 and IFN- $\gamma$ by ELISAs. 
A

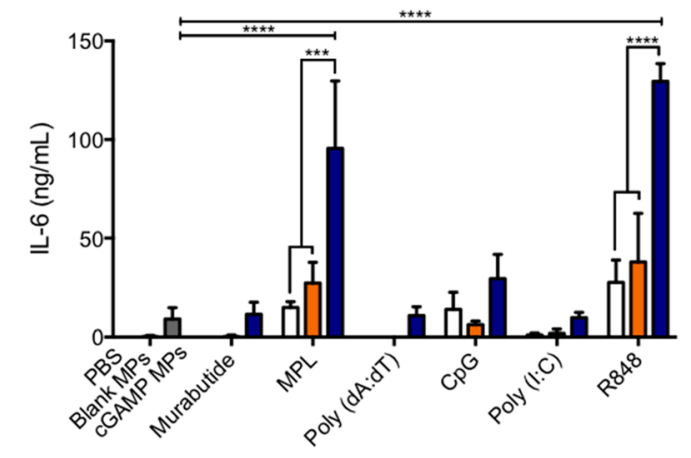

C

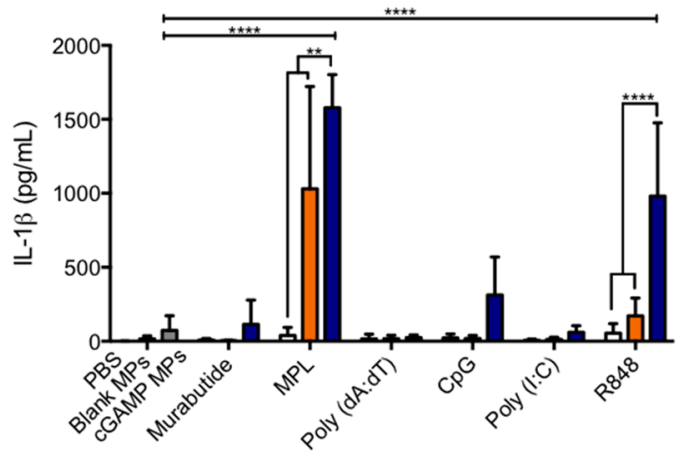

E

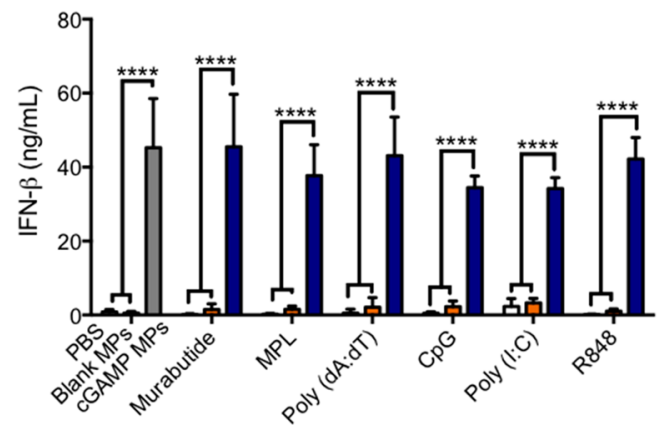

B

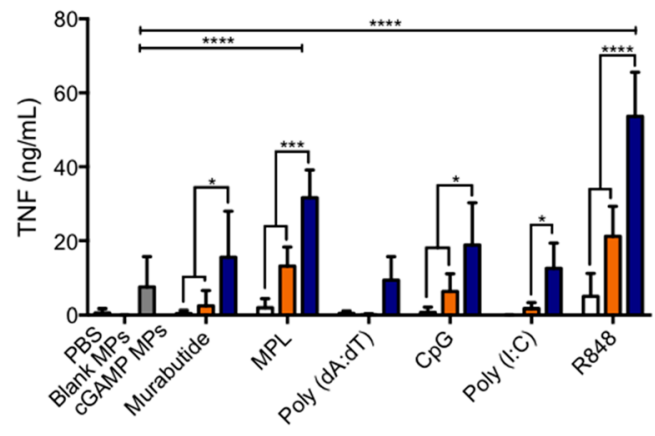

D

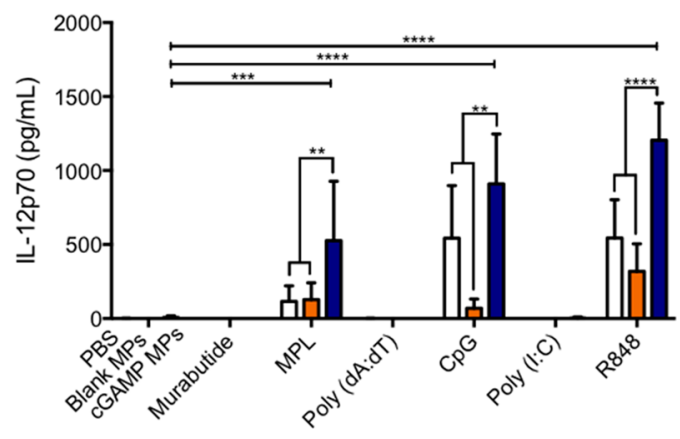

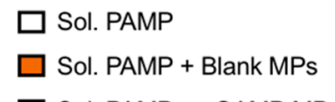

Sol. PAMP + cGAMP MPS

Figure 1. Combined treatment of cGAMP microparticles (MPs) with various soluble pathogen-associated molecular patterns (PAMPs). Bone marrow derived dendritic cells (BMDCs) from C57BL/6 mice were treated with soluble (Sol.) murabutide $(10 \mu \mathrm{g} / \mathrm{mL}), \mathrm{MPL}(1 \mu \mathrm{g} / \mathrm{mL})$, poly (dA:dT) $(10 \mu \mathrm{g} / \mathrm{mL}), \mathrm{CpG}(1 \mu \mathrm{g} / \mathrm{mL})$, poly $(\mathrm{I}: \mathrm{C})(10 \mu \mathrm{g} / \mathrm{mL})$, or R848 $(0.01 \mu \mathrm{g} / \mathrm{mL})$ alone, or in combination with $1 \mu \mathrm{g} / \mathrm{mL} \mathrm{cGAMP} \mathrm{MPs}$ or an equivalent amount of blank MPs. The left-most data group displayed in each panel includes a PBS control (checkered bar), an equivalent amount of blank MPs to the cGAMP MPs (black bar), and $1 \mu \mathrm{g} / \mathrm{mL}$ cGAMP MPs only (gray bar). Cell supernatants were collected after $22 \mathrm{~h}$ and were analyzed for (A) IL-6, (B) TNF, (C) IL-1 $\beta$, (D) IL-12p70, and (E) IFN- $\beta$ ( $n=4 \pm$ SEM, $* p<0.05$, ** $p<0.01, * * * p<0.001, * * * * p<$ $0.0001)$.

Statistical Analysis. All data were analyzed using GraphPad Prism software. Group comparisons were analyzed using one-way analysis of variance (ANOVA), with differences between groups assessed using Tukey's post hoc test. A p-value of less than 0.05 was considered statistically significant. Data are reported as indicated in figure captions.

\section{RESULTS AND DISCUSSION}

Previously, our laboratory has demonstrated that Ace-DEX MPs encapsulating cGAMP were safe and potent inducers of type-I interferon (IFN), and vaccination using a cGAMP MP adjuvant protected mice against a lethal influenza challenge with dose sparing over soluble cGAMP. ${ }^{22}$ In that work, cGAMP MPs alone generated high levels of IFN- $\beta$, IL-6, and
$\mathrm{TNF}^{22}$ which are all key contributors in generating a robust immune response; however, cGAMP MPs lacked the ability to produce IL- $1 \beta$ and IL-12p70, which are critical during viral infections and play pivotal roles in generating adaptive immune responses. ${ }^{43,44}$ Additionally, recombinant IL-1 $\beta^{31,45,46}$ and IL$12^{47,48}$ have been used successfully as vaccine adjuvants in preclinical model systems. Therefore, in our current study, we aimed to identify a combination of PAMP adjuvants that can elicit a broader cytokine profile, including IL- $1 \beta$ and IL-12p70. Murine BMDCs were treated with cGAMP MPs alone or in combination with a variety of soluble PAMPs. We tested several PAMPs that target TLRs either on the plasma membrane (FDA-approved TLR4 agonist MPL) or phagosomal membranes (TLR3 agonist poly I:C, TLR7/8 agonist 
Table 1. Encapsulation Efficiencies (EE) of cGAMP, Resiquimod (R848), or a Combination of Both (cGAMP/ R848) within Acetalated Dextran (Ace-DEX) or Poly Lacticco-glycolic Acid (PLGA) Microparticles by Emulsion and Electrospray

\begin{tabular}{clcc}
$\begin{array}{c}\text { fabrication } \\
\text { technique }\end{array}$ & \multicolumn{1}{c}{ microparticles } & $\begin{array}{c}\text { cGAMP EE } \\
(\%)\end{array}$ & $\begin{array}{c}\text { R848 EE } \\
(\%)\end{array}$ \\
emulsion & cGAMP Ace-DEX & 52 & -- \\
& cGAMP PLGA & 3 & -- \\
& R848 Ace-DEX & -- & 8 \\
& R848 PLGA & -- & 0 \\
electrospray & cGAMP/R848 Ace-DEX & 25 & 8 \\
& cGAMP Ace-DEX & 94 & -- \\
& R848 Ace-DEX & -- & 86 \\
& cGAMP/R848 Ace-DEX & 83 & 75 \\
& cGAMP/R848 PLGA & 74 & 67 \\
\hline
\end{tabular}

R848, or TLR9 agonist CpG). Poly(dA:dT), which is detected by cytosolic DNA sensors, and NOD2 agonist murabutide were also evaluated in combination with cGAMP MPs. Among the group of PAMPs examined, MPL and R848 showed the greatest impact on cytokine responses (Figure 1). In combination with cGAMP MPs, both PAMPs induced significant levels of IL- $1 \beta$ and IL-12p70, which were not achieved with either cGAMP MPs or soluble PAMPs alone. Furthermore, the combinations significantly enhanced production of IL-6 and TNF compared to each stimulus on their own.
MPL in combination with cGAMP MPs demonstrated significant increases in cytokine production;, however, R848 was overall more potent than MPL. R848 is an imidazoquinoline agonist of TLR7/8 with potent myeloid differentiation primary response gene 88 (MyD88)-dependent transcriptional activation of NF- $\kappa \mathrm{B}$ pathways. The use of $\mathrm{R} 848$ in a microparticulate vaccine is relevant to humans, as TLR7 and TLR8 are predominantly expressed in human APCs such as plasmacytoid dendritic cells, myeloid dendritic cells, and monocytes. $^{49}$ R848 has previously been shown to have additive effects in vivo with a MyD88-independent TLR3 agonist, ${ }^{50}$ which suggests that R848 and cGAMP would have significant activity in vivo.

In addition to R848 eliciting the most robust responses, it was chosen for further study for several other reasons. First, very low doses were required ( 100 -fold lower than MPL). Second, we have previously demonstrated that the acidsensitive Ace-DEX MPs are highly effective for delivering R848. ${ }^{29,30,51,52}$ Finally, while other groups have previously demonstrated that combinations of cGAMP with the TLR9 agonist $\mathrm{CpG}$ result in IL-12 production and improved in vivo vaccine outcomes, ${ }^{53,54}$ and liposomal c-di-GMP (another $\mathrm{CDN}$ ) with MPL led to improved humoral and cellular immunity, ${ }^{10}$ combinations of CDNs with R848 remained unexplored.

To formulate cGAMP and R848 into Ace-DEX MPs, we took several approaches including emulsion and electrospray processes. Emulsion-based, solvent evaporation MPs were formulated to determine if encapsulation efficiencies (EEs) of cGAMP and R848 would remain constant when combining the
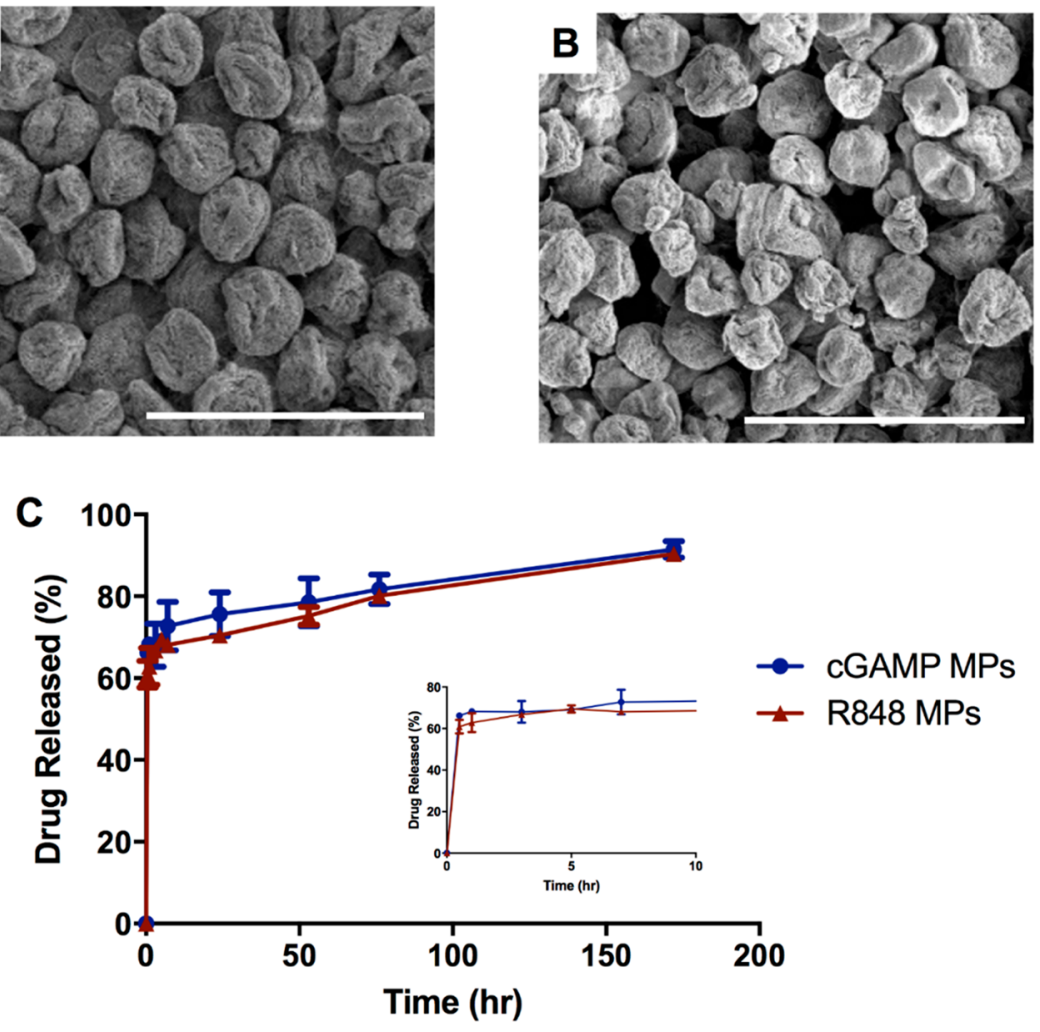

Figure 2. Morphology and release kinetics of cGAMP and R848 individually encapsulated in acetalated dextran microparticles (Ace-DEX MPs) made by electrospray. Scanning electron micrographs of (A) cGAMP Ace-DEX MPs and (B) R848 Ace-DEX MPs. Scale bars $=4 \mu \mathrm{m}$. (C) Release profiles of cGAMP and R848 individually encapsulated into acetalated dextran MPs were conducted at $\mathrm{pH}$ 7.4. Data are presented as mean \pm SEM $(n=3)$. 
A

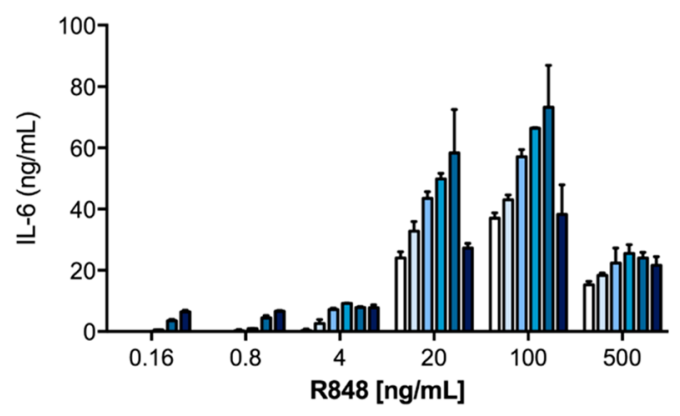

C

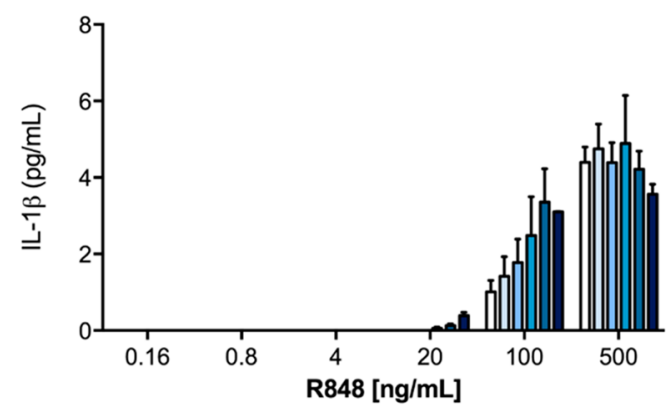

E

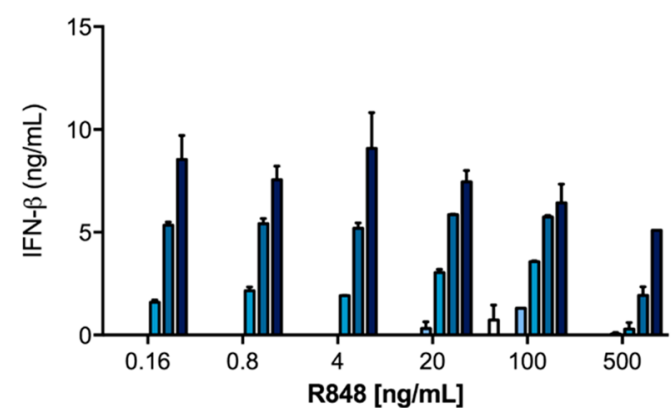

B

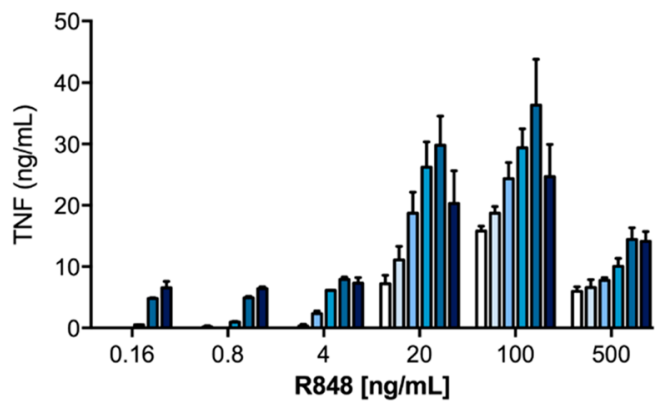

D

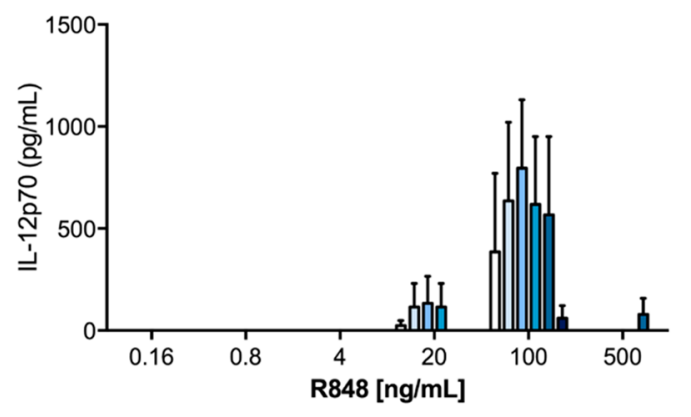

cGAMP [ng/mL]

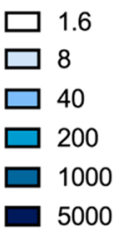

Figure 3. Optimal ratio determination for cGAMP and resiquimod (R848) microparticles. Bone marrow derived dendritic cells from C57BL/6 mice were treated for $22 \mathrm{~h}$ with indicated concentrations of resiquimod (R848) and cGAMP, both of which were individually encapsulated within acetalated dextran (Ace-DEX) microparticles. Supernatants were analyzed for (A) IL-6, (B) TNF, (C) IL-1 $\beta$, (D) IL-12p70, and (E) IFN- $\beta$. Data are presented as mean $\pm \operatorname{SEM}(n=4)$

two molecules within the same formulation. We also examined the clinically relevant biodegradable polymer, PLGA. Because we have previously formulated various PAMPs into PLGA $(85: 15) \mathrm{MPs}^{22,26}$ for comparative purposes we chose to use this polymer grade again. The MPs demonstrated a poor 3\% encapsulation efficiency (EE) of cGAMP and were unable to encapsulate R848 (Table 1). Conversely, the acid-sensitive biopolymer, Ace-DEX, demonstrated modestly higher EEs of cGAMP (52\%) and R848 (8\%) in singularly encapsulated particles. These EEs for R848 were consistent with previous work reported by our lab $\mathrm{l}^{29,30,52}$ and are likely driven by loss of the cargo to the external aqueous phase of the emulsion. ${ }^{55}$

Formulating cGAMP MPs and R848 MPs through coaxial electrospray yielded significantly higher EEs compared to MPs formulated through the emulsion-based method. Scanning electron micrographs demonstrated spherical and fairly monodispersed electrospray MPs with EEs of 94 and 86\% for cGAMP and R848, respectively (Figure 2). The release kinetics of cGAMP and R848 MPs were very similar, with a large burst release due to drug diffusion occurring within the first $30 \mathrm{~min}$ of incubation, followed by a sustained release for the duration of the 1 week experiment (Figure 2C). This is similar to previously reported cGAMP and R848-loaded AceDEX MP formulations. ${ }^{22,27}$

We next set out to identify the ratio of cGAMP and R848 that yielded optimal biological activity, in order to inform target weight loadings for coencapsulated particles. BMDCs were treated for $24 \mathrm{~h}$ with the indicated doses of individually encapsulated cGAMP and R848 Ace-DEX MPs, and supernatants were then assayed for cytokine production (Figure 3, heat mapped in Supplementary Figure S1). The trends in the IL-6, TNF, IL-1 $\beta$, and IL-12p70 data suggest that the optimal responses occurred at a 10:1 weight-to-weight $(\mathrm{w} / \mathrm{w})$ ratio of cGAMP to R848, which was therefore used in subsequent particle formulations. Decreased cytokine production at higher cGAMP concentrations was also illustrated in our previous work using cGAMP MPs alone. ${ }^{22}$

In our previous work, multiple analytes have been loaded into Ace-DEX MPs via double-emulsion and coaxial electrospray, $^{23,52,56}$ and those methods were also employed to coformulate cGAMP and R848. The emulsion-based combination MPs of cGAMP and R848 resulted in a drop from 52 to 

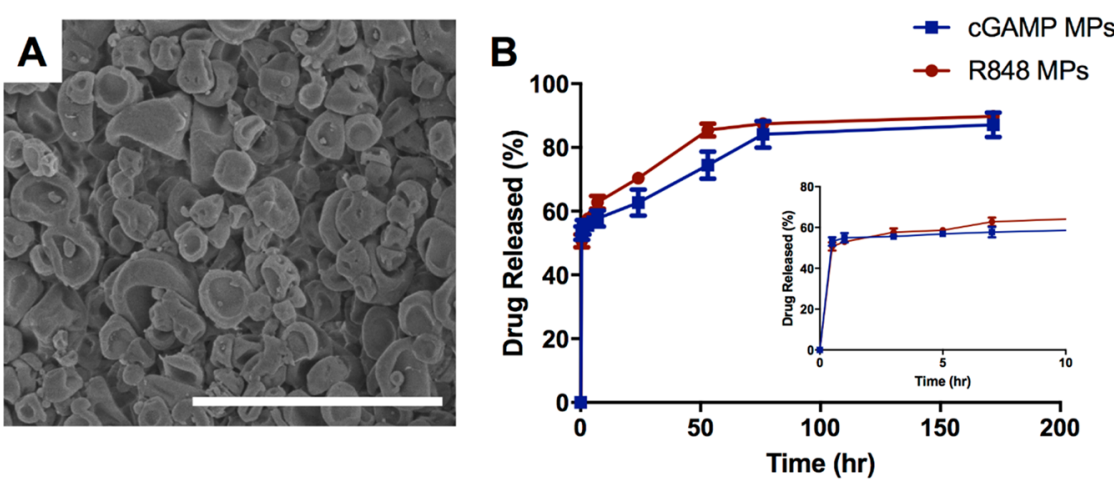

C

\begin{tabular}{ccc}
\hline Electrospray Particle Formulation & $\begin{array}{c}\text { cGAMP Loading } \\
(\mu \mathrm{g} / \mathrm{mg})\end{array}$ & $\begin{array}{c}\mathbf{R} 848 \text { Loading } \\
(\mu \mathrm{g} / \mathrm{mg})\end{array}$ \\
\hline cGAMP/R848 Ace-DEX MPs & 8.3 & 0.75 \\
\hline
\end{tabular}

Figure 4. Morphology and release kinetics of combination microparticles made by electrospray. (A) Scanning electron micrographs of acetalated dextran microparticles encapsulating a combination of cGAMP and resiquimod (cGAMP/R848 Ace-DEX MPs). Scale bar indicates $4 \mu \mathrm{m}$. (B) Release profiles of cGAMP and R848 within combination MPs conducted at pH 7.4. (C) Drug loading of cGAMP and R848 ( $\mu \mathrm{g} / \mathrm{mg})$. Data are presented as mean $\pm \operatorname{SEM}(n=3)$.

$25 \%$ EE of cGAMP (Table 1), essentially encapsulating half the drug of the individually encapsulated formulation. The EE of R848 persisted at a low $8 \%$. Although certain parameters can be modified to enhance the EEs of polymeric MPs formed through solvent evaporation, a target ratio is extremely difficult to repeatedly formulate. ${ }^{57}$ Furthermore, emulsion-based solvent evaporation methods are a batch process, which can result in inconsistencies in loading, a high cost for scale-up, and be difficult in aseptic environments, thus limiting the potential for clinical applications. ${ }^{58}$ More recently, a dual impinger technology has become increasingly popular, in which two phases are pumped together simultaneously with turbulent flow to generate particles; however, an external aqueous phase is still a driving force for poor EE. ${ }^{55}$ To increase the EE of cGAMP and R848 within that of Ace-DEX MPs and generate particles using a more scalable process, electrospray was utilized to formulate particles used in all subsequent experiments.

Combination MPs containing cGAMP and R848 (10:1 ratio) in the same particle were coaxially electrosprayed and displayed an erythrocyte-like morphology (Figure 4). This differed from the more spherical singularly encapsulated cGAMP or R848 MPs (Figure 2). The release profile of each adjuvant was very similar to the release of the individually encapsulated particles with a large burst occurring within the first $30 \mathrm{~min}$ of incubation, followed by a sustained release for the duration of the experiment. The release kinetics following the burst release of the combination Ace-DEX MPs was faster in a $\mathrm{pH} 7.4$ solution than that of the singularly encapsulated particles (Figure 2C). The EE decreased for both PAMPs when coencapsulated via electrospray, but overall, the EEs were significantly greater than for coencapsulation MPs formed through emulsion or either method with PLGA (Table 1). Drug loading of the combination MPs was measured at $8.3 \mu \mathrm{g} /$ $\mathrm{mg}$ of cGAMP and $0.75 \mu \mathrm{g} / \mathrm{mg}$ of R848, very close to the target ratio of 10:1 (Figure 4C). To ensure that both compounds could be encapsulated into a single MP, multiple fluorophores were sprayed and analyzed using flow cytometry (Supplemental Figure S.2). This analysis demonstrated that all three fluorescent small molecules were distributed throughout the entire particle population at high levels.

The ability to target a specific drug ratio and create a particle population with both drugs at these ratios with minimal drug loss is a highly advantageous formulation strategy. Once the ratio was selected and MPs made, the combination MPs were analyzed in comparison to singularly encapsulated particles delivered together in vitro (Figure 5). Comparing the separate and combination particles, our data indicated that there is an advantage to having the adjuvants within the same particle.

We wanted to then evaluate if the differences were dependent on the polymer chosen, so we compared AceDEX versus PLGA MPs. Ace-DEX is an ideal delivery vehicle for adjuvants with intracellular targets such as STING and TLR7/8 because of its acid-sensitive degradation kinetics. ${ }^{19}$ Once an Ace-DEX particle is phagocytosed by an phagocytic cell, $^{23}$ the particle will rapidly degrade within the low $\mathrm{pH}$ environment of the lysosome. ${ }^{38}$ According to the release profiles in Figures 2 and 4, both water-soluble PAMPs take over 7 days to achieve maximum release at $\mathrm{pH} 7.4$; however, in the lower $\mathrm{pH}$ environment of the lysosome, maximum release should occur in only a few hours or less. ${ }^{22}$ While the burst release observed for R848 and cGAMP is not ideal, these are both water-soluble molecules that may be diffusing rapidly through the hydrophobic polymeric formulation to reach the external aqueous environment. Indeed, burst release is not uncommon with water-soluble molecules encapsulated within hydrophobic microparticle formulations. ${ }^{59}$ Since PLGA is used in approximately 15 FDA-approved drug delivery formulations, ${ }^{60}$ we evaluated the innate immune response of Ace-DEX or PLGA MPs encapsulating cGAMP and R848 (cGAMP/ R848 PLGA MPs; Table 1, Figure 6). cGAMP/R848 Ace-DEX MPs elicit robust IL-6, TNF, IL- $1 \beta$, IL-12p70, and IFN- $\beta$ responses, indicating that Ace-DEX is an ideal drug carrier for vaccine adjuvant systems. Additionally, cGAMP/R848 AceDEX MPs result in significantly greater induction of TNF and IFN- $\beta$ compared to cGAMP/R848 PLGA MPs, which aligns with our previous work where we demonstrated that cGAMP MPs consisting of Ace-DEX elicited more potent immune responses than PLGA as well as liposomes. ${ }^{22}$ It is theorized 
A

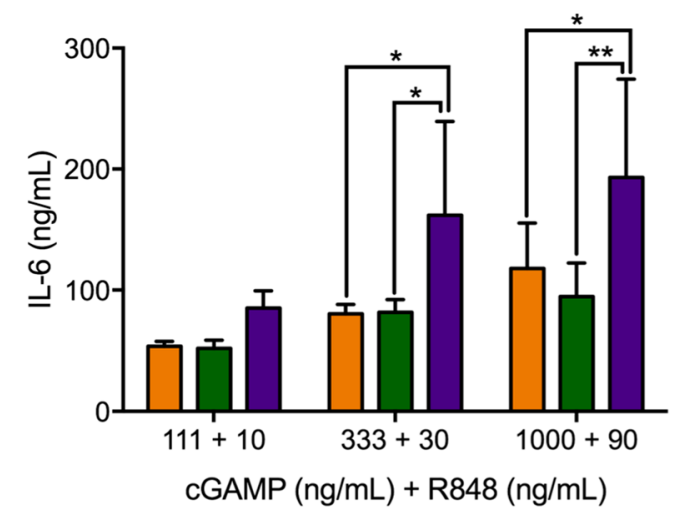

C

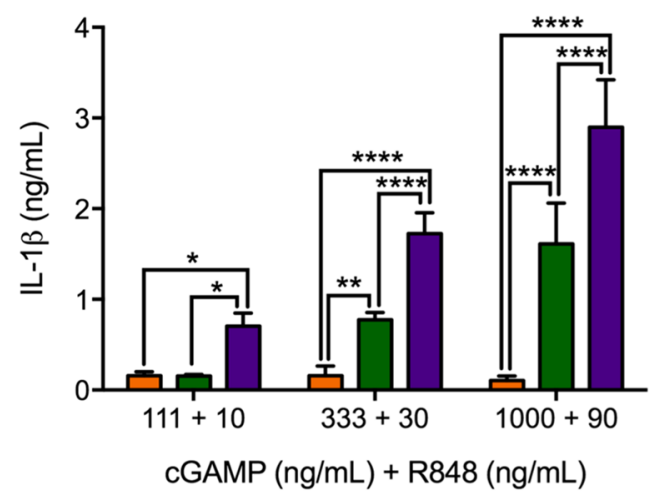

E

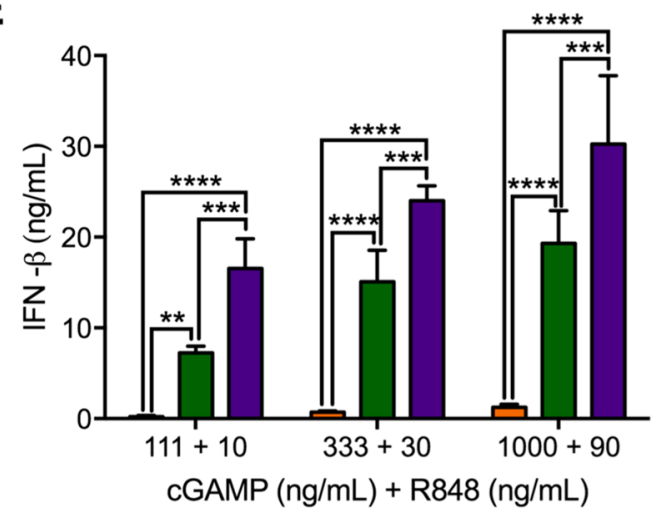

B

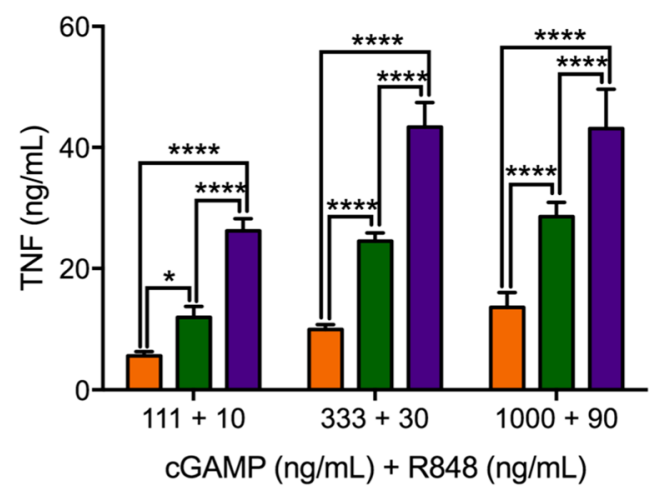

D

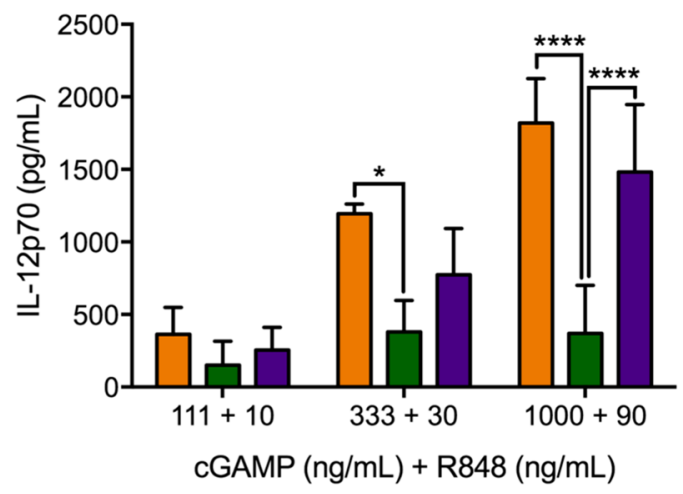

$\square$ Sol. cGAMP + Sol. R848
cGAMP MPs + R848 MPs
cGAMP/R848 MPs

Figure 5. Coencapsulation of cGAMP and R848 in acetalated dextran microparticles (Ace-DEX MPs) compared to individual encapsulation. Bone marrow derived dendritic cells from C57BL/6 mice were treated with indicated concentrations of cGAMP and R848, respectively, delivered as soluble (Sol.) drugs, encapsulated in separate Ace-DEX MPs (cGAMP MPs + R848 MPs) or coencapsulated within the same Ace-DEX MPs (cGAMP/R848 MPs). After $22 \mathrm{~h}$, supernatants were harvested and analyzed for (A) IL-6, (B) TNF, (C) IL-1 $\beta$, (D) IL-12p70, and (E) IFN- $\beta$ ( $n=$ $4 \pm$ SEM, $* p<0.05, * * p<0.01, * * * p<0.001, * * * * p<0.0001)$

that the slow degradation kinetics of PLGA and minimal $\mathrm{pH}$ sensitivity of the polymer prohibited cGAMP from releasing quick enough within the lysosome to induce potent cytokine production.

To better characterize the promising results we observed in vitro, we next evaluated the formulation in vivo. Initially we evaluated the acute toxicity of the combination MPs. cGAMP/ R848 Ace-DEX MPs (200 ng of cGAMP and $18 \mathrm{ng}$ of R848) were injected intramuscularly (i.m), and mice were monitored for 7 days to assess toxicological outcomes including body weight, body temperature, body condition (a composite of activity, weight loss, body temperature, hydration, physical appearance, and appetite), and survival (Supplementary Figure S3). No acute toxicity, adverse events, or injection site reactogenicity were observed.

After acute toxicity of the formulation was evaluated, mice were vaccinated against the model antigen OVA with various formulations (Table S1). Blank Ace-DEX and PLGA MP controls were included, as well as alum, a clinically relevant adjuvant which served as a positive control. On day 28 , serum was collected and analyzed for antibody titers against OVA (Figure 7). In comparing the formulations, no other MP group resulted in a greater than 2.5 -fold increase in total IgG with respect to cGAMP MPs except for cGAMP/R848 Ace-DEX 
A

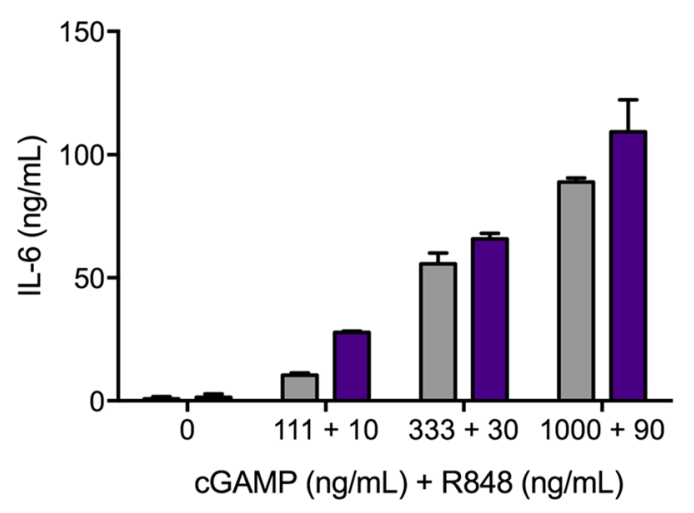

C

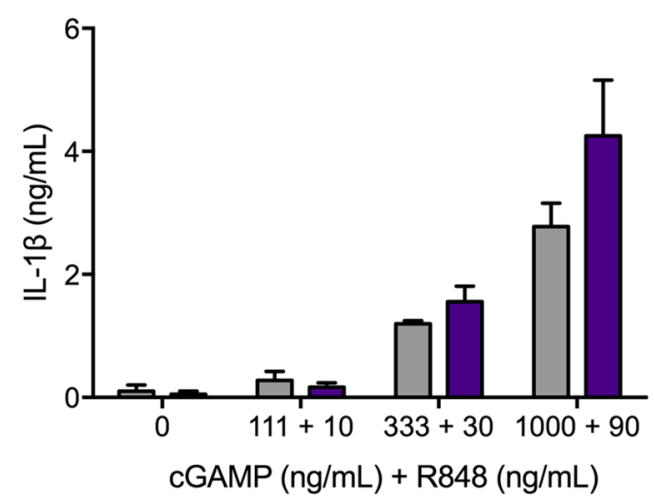

E

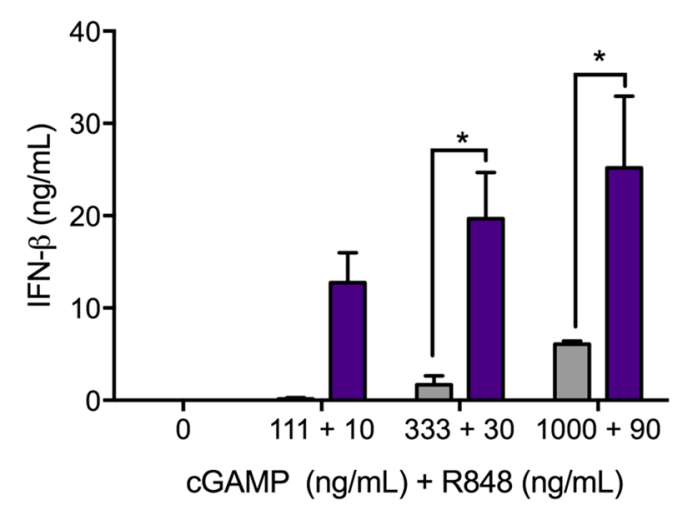

B

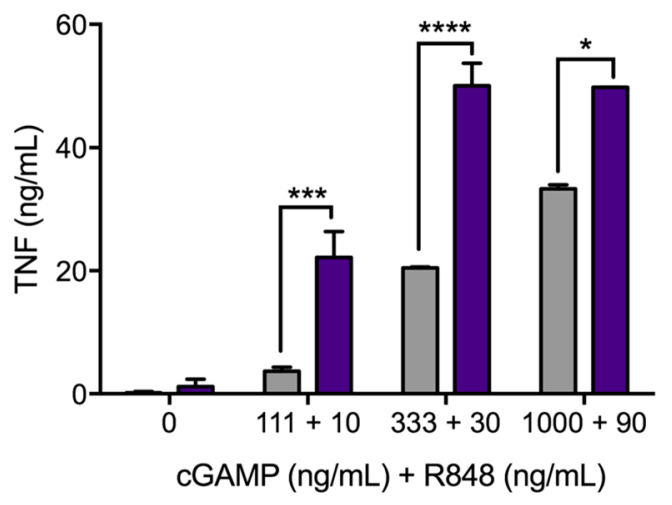

D

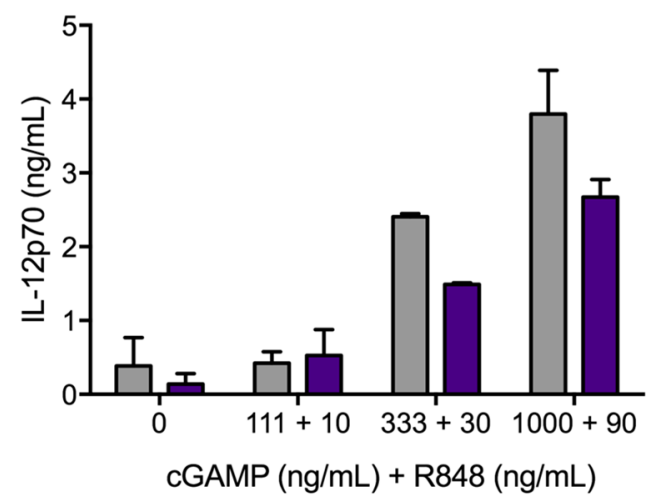

Figure 6. Cytokine profiles of combination cGAMP/R848 acetalated dextran (Ace-DEX) or poly(lactic-co-glycolic acid) (PLGA) MPs. Bone marrow derived dendritic cells from C57BL/6 mice were treated with indicated concentrations of cGAMP and R848, respectively, coencapsulated within Ace-DEX or PLGA MPs. After $22 \mathrm{~h}$, supernatants were harvested and analyzed for (A) IL-6, (B) TNF, (C) IL-1 $\beta$, (D) IL-12p70, and (E) IFN $-\beta(n=4 \pm$ SEM $* p<0.05, * * p<0.01, * * * p<0.001, * * * * p<0.0001)$.

MPs, which resulted in over a 7.5-fold increase. Strikingly, delivery of cGAMP/R848 Ace-DEX MPs resulted in antibody titers 10-fold higher than those obtained using combination cGAMP/R848 PLGA MPs, supporting the in vitro bioactivity results using the Ace-DEX MP formulation. Combination cGAMP/R848 Ace-DEX MPs also induced higher total IgG titers compared to individually encapsulated cGAMP and R848 Ace-DEX MPs delivered either alone or together. This complements results observed by Kasturi et al. ${ }^{57}$ and demonstrates the importance of having multiple adjuvants within the same particle for humoral responses. These results suggest that the antibody titers produced from the combination MPs are not simply an additive effect between the two adjuvants but rather a synergistic effect. ${ }^{61}$

While total IgG generation reveals the humoral response to vaccination, IgG1 and $\operatorname{IgG} 2 \mathrm{c}$ subtypes are indicative of the strength of the inflammatory process by Th2- and Th1-skewed responses, respectively. ${ }^{62}$ For certain pathogens, it is important to achieve more balanced Th1/Th2 humoral immunity, because both responses are critical for successful clearance. As with total IgG, combination cGAMP/R848 Ace-DEX MPs induced the highest overall IgG1 and IgG2c titers. Consistent with our previously reported findings, all formulations including cGAMP induced significant levels of IgG2c and 
A

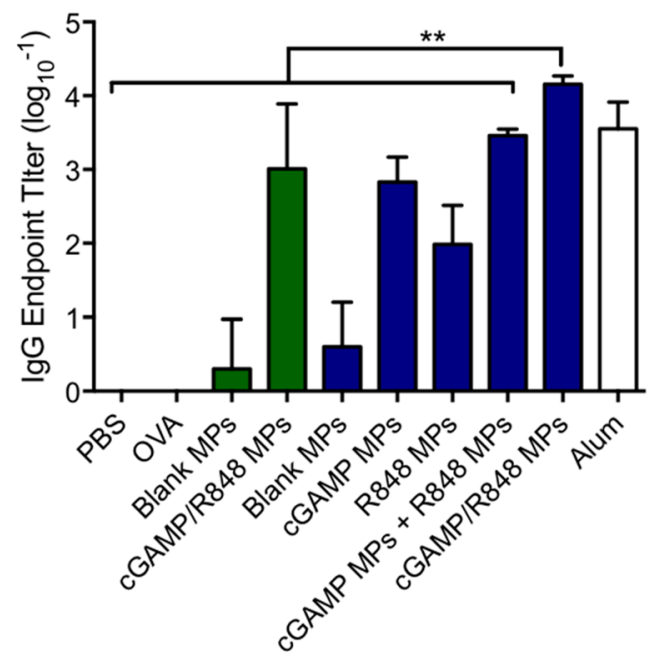

B

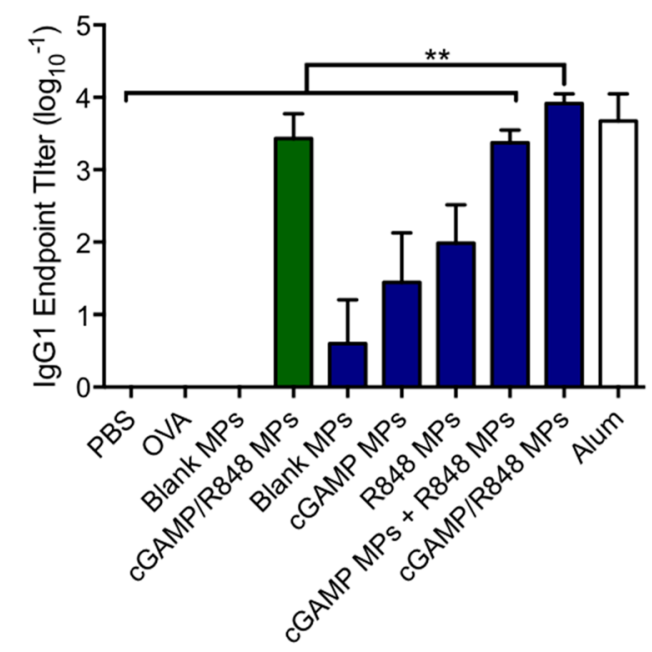

Ace-DEX MPs

D

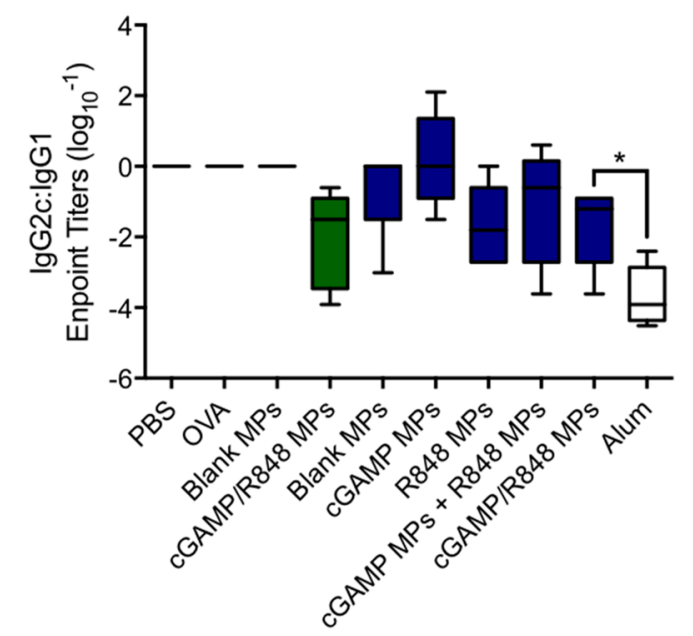

Figure 7. Comparison of antibody responses induced by cGAMP and R848 delivered via acetalated dextran (Ace-DEX) or poly(lactic-co-glycolic acid) (PLGA) microparticles (MPs). C57BL/6 mice were immunized intramuscularly on days 0 and 21 . All groups received $10 \mu \mathrm{g}$ of OVA, except the PBS group. OVA was given alone or in combination with cGAMP (200 ng) and R848 (18 ng) delivered in single-loaded or dual-loaded AceDEX or PLGA MPs, blank Ace-DEX or PLGA MPs, or alum. On day 28, serum was collected and analyzed for ovalbumin (OVA)-specific (A) total $\mathrm{IgG}$, (B) IgG1, and (C) IgG2c as well as (D) the ratio between IgG2c and IgG1 isotypes $\left(n=4-5 \pm\right.$ SEM, ${ }^{*} p<0.05$, ** $p<0.01$, *** $p<0.001$, $* * * * p<0.0001)$. Data are presented as $n=5 \pm$ SD.

resulted in a near even ratio of $\operatorname{IgG} 2 \mathrm{c} / \mathrm{IgG} 1$, indicating a balanced Th1/Th2 humoral response. ${ }^{22}$ This is in stark contrast to alum, which showed a profound bias toward IgG1, indicating potent Th2 polarization. The IgG1 responses in groups with combination adjuvant systems (coencapsulated and singularly encapsulated combination) were larger than the responses seen with cGAMP alone, demonstrating that Th2type responses can be influenced by dual activation of the immune system (Figure 7). Vaccination with cGAMP/R848 Ace-DEX MPs elicited the largest production of IgG1 compared to all other formulations, indicating that Th2 skewing could benefit from coencapsulation and Ace-DEX degradation kinetics. ${ }^{24}$ Th1 skewing has been implicated in long-lasting immunity, which is pivotal for a successful vaccine. ${ }^{63}$ IgG2c production seemed to be independent of coencapsulation, as vaccination with cGAMP/R848 Ace-DEX
MPs or the combination of cGAMP MPs and R848 MPs resulted in similar titers. These results shed some light on how adjuvant systems can induce more balanced humoral immune skewing, which is critical for broad acting vaccines.

Th1 and Th2 skewing via IgG subtypes is not fully indicative of cellular and humoral recognition of antigens, and thus, CD8+ and CD4+ T cell activities were assessed. Splenocytes were collected on day 42 and restimulated with the CD8+ restricted OVA T cell epitope SIINFEKL for the assessment of antigen-specific IFN- $\gamma$ or IL-2 producing $\mathrm{T}$ cells by ELISpot (Figure 8A,C) or whole OVA protein, for the total production of IFN- $\gamma$ and IL-2 in culture supernatants as assessed by ELISAs (Figure 8B,D). The combination cGAMP/R848 AceDEX MPs induced significant IFN- $\gamma$ and IL-2 production compared to controls containing OVA alone, OVA plus either R848 MPs, or alum (Figure 8B,D). Interestingly, while the 
A

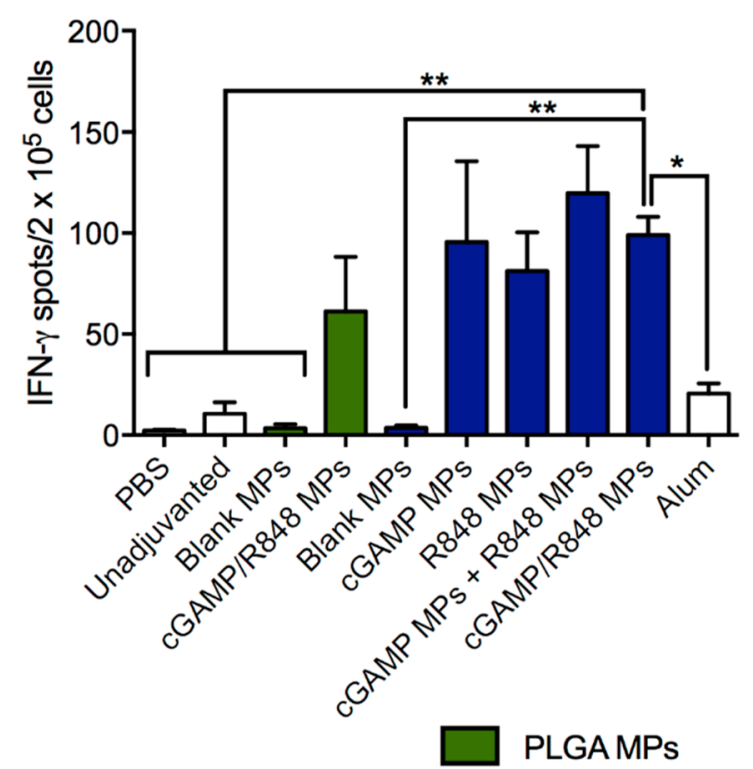

C

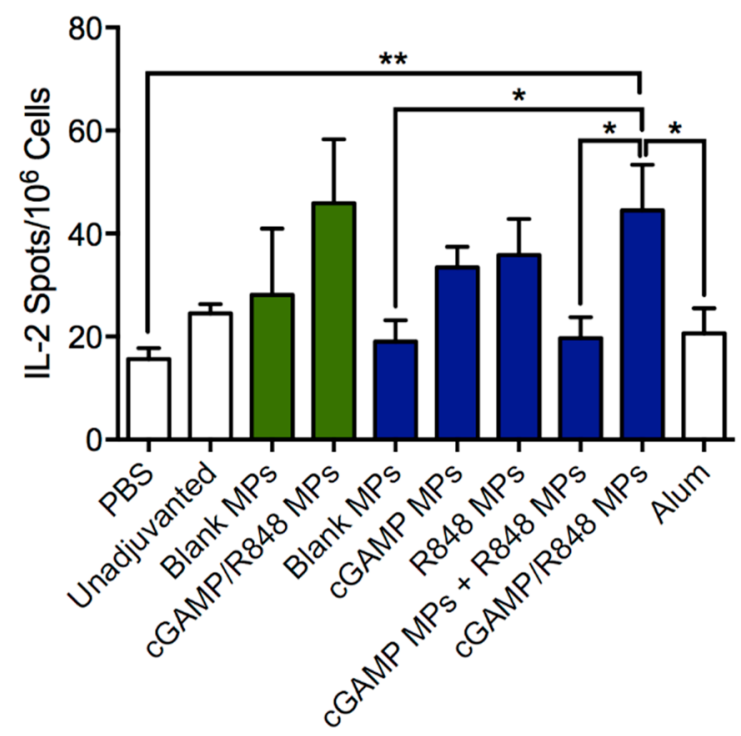

B

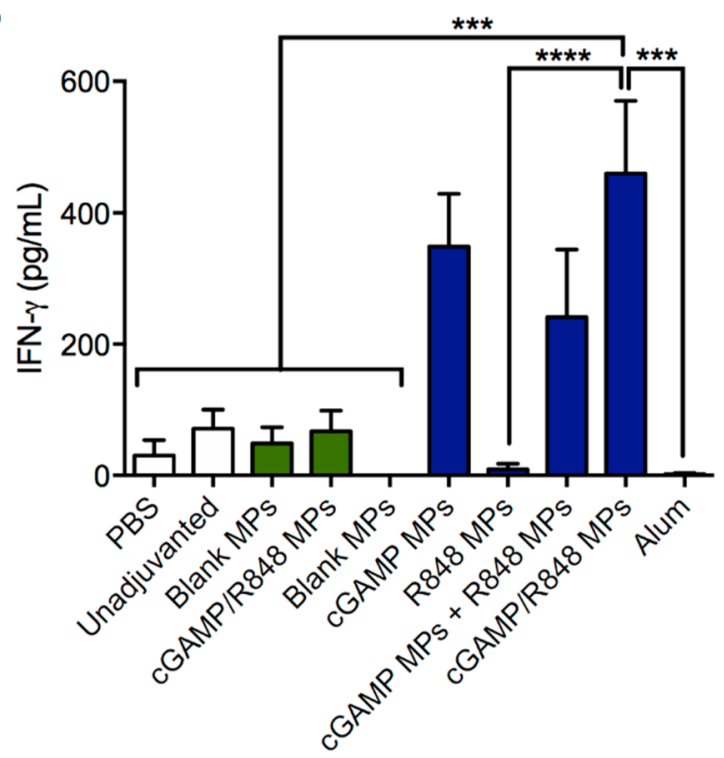

Ace-DEX MPs

D

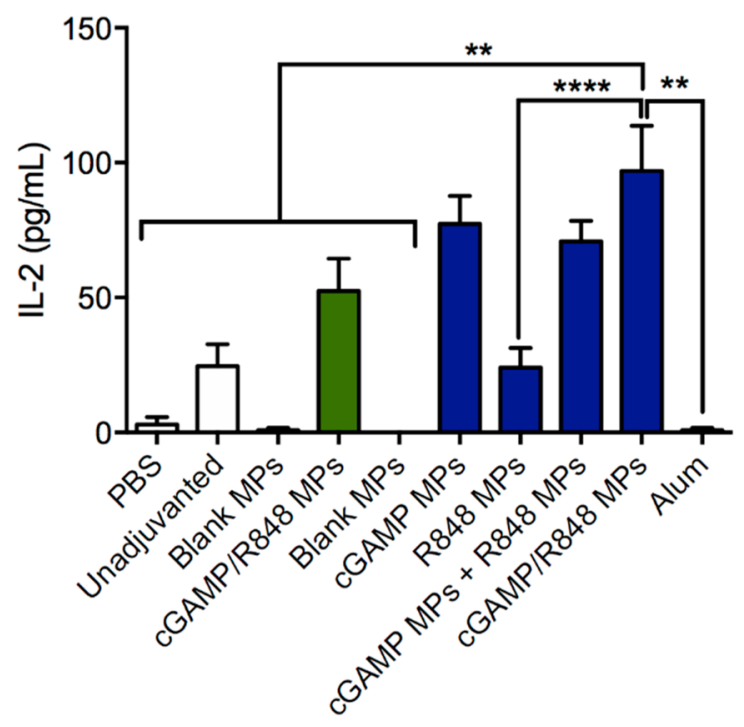

Figure 8. Comparison of T cell responses induced by cGAMP and R848 delivered via acetalated dextran (Ace-DEX) or poly(lactic-co-glycolic acid) (PLGA) microparticles (MPs). C57BL/6 mice were immunized intramuscularly on days 0,21 , and 35 . All groups received $10 \mu \mathrm{g}$ of OVA, except the PBS group. OVA was given alone or in combination with cGAMP (200 ng) and R848 (18 ng) delivered in single-loaded or dual-loaded AceDEX or PLGA MPs, blank Ace-DEX or PLGA MPs, or alum. On day 42, mice were sacrificed and (A) IFN- $\gamma$ and (C) IL-2 ELISpots were performed on splenocytes restimulated with SIINFEKL peptide $(10 \mu \mathrm{g} / \mathrm{mL})$ for $36 \mathrm{~h}$. Alternatively, splenocytes were stimulated with $10 \mu \mathrm{g} / \mathrm{mL}$ ovalbumin (OVA) protein for $36 \mathrm{~h}$. Supernatants were analyzed for (B) IFN $-\gamma$ and (D) IL-2 by ELISA $(n=4-5 \pm$ SD, $* p<0.05$, **p $\leftarrow 0.01$, $* * * p<0.001, * * * * p<0.0001)$.

cGAMP/R848 PLGA MPs had comparable OVA-specific Tcell responses compared to cGAMP/R848 Ace-DEX MPs by ELISpot (not statistically different), the Ace-DEX MP formulation induced significantly greater IFN- $\gamma$ and IL-2 responses (as observed by ELISA), indicating a more potent antigen-specific $\mathrm{T}$ cell activation (Figure 8B,D). These differential responses indicate that perhaps the CD8+ activity of the vaccine is not solely dependent upon coencapsulation of adjuvants but also dependent on the timing of adjuvant delivery, which agrees with our previous work. ${ }^{20,24}$ Also, IFN- $\gamma$ production was measured following whole OVA stimulation of splenocytes, indicating that the CD4+ epitope was successfully recognized. Together, these results indicate that both coencapsulation of adjuvants and degradation kinetics of the vehicle can enhance antigen-specific $\mathrm{T}$ cell responses, similar to results documented by Savelkoul et al. ${ }^{64}$ and Chen et al., ${ }^{24}$ respectively.

Several previous reports have examined codelivery of multiple TLR agonist adjuvants in PLGA particles fabricated through single- or double-emulsion procedures. ${ }^{57,65-71}$ These reports agree with our observations using cGAMP/R848 AceDEX MPs, in that coencapsulation of TLR agonists provided either dose sparing ${ }^{66}$ and/or enhancement of biological 
responses $^{65-67,69}$ compared to soluble TLR agonist combinations.

We also determined experimentally that delivery of PAMPs in the same MPs induced superior responses both in vitro and in vivo when compared to the same dose of PAMPs delivered in separate MPs. One likely explanation for this observation is that coencapsulated agonists will always engage their respective PRRs in the same cell (cis-engagement), allowing for PRR cross-talk and enhanced immune activation. Conversely, when PAMPs are delivered in separate particles, both particle types need to be delivered intracellularly in order for PRR crosstalk to occur (trans-engagement). The likelihood of this happening is dependent upon both particle location and the phagocytic capacity of the cell, leading to a more variable response. This cis- vs trans-engagement of receptors is poorly studied in the context of innate immunity but may be important for optimal biological responses, as PRR cross-talk plays a central role in innate immunity and allows the generation of a pathogenspecific immune response. ${ }^{35}$ In addition, others have shown that the combination of coencapsulated PAMPs had a stronger adjuvant effect than the delivery of individually encapsulated PAMPs. ${ }^{57,71}$ Therefore, our work and the work of others strongly supports that coengagement of multiple PRRs is able to generate potent humoral and cellular responses. ${ }^{57,67,70,71}$

Taken together, Ace-DEX MPs containing cGAMP and R848 stand as a novel and efficacious formulation for vaccine development. Novel and potent adjuvant systems are needed to develop efficacious vaccines that generate balanced immune responses. While the MPL and alum adjuvant system (AS04) provides a balanced immune response, cGAMP/R848 AceDEX MPs offer a unique delivery vehicle that can passively target APCs and deliver adjuvants extremely quickly once internalized. The doses used within these experiments were on the nanogram scale, also offering a dose sparing alternative to traditional adjuvant vaccine systems. cGAMP/R848 Ace-DEX MPs represent a potent adjuvant system that can be administered at extremely low doses with strong immune responses for vaccine applications.

\section{ASSOCIATED CONTENT}

\section{S Supporting Information}

The Supporting Information is available free of charge on the ACS Publications website at DOI: 10.1021/acs.molpharmaceut.8b00579.

Supplementary Table 1: vaccine formulations for in vivo studies; Supplementary Figure 1: heat map of cytokines induced by treatment with combination cGAMP and R848 acetalated dextran microparticles (Ace-DEX MPs); Supplementary Figure 2: flow cytometry analysis of acetalated dextran microparticles (Ace-DEX MPs) encapsulating various fluorophores; Supplemental Figure 3: characterization of toxicity outcomes in vivo following acetalated dextran microparticle (Ace-DEX MP) administration (PDF)

\section{AUTHOR INFORMATION}

\section{Corresponding Author}

*E-mail: ainsliek@email.unc.edu; Mailing Address: Kristy Ainslie, 125 Mason Farm Road, Chapel Hill, NC 27599, United States.

\section{ORCID}

Monica M. Johnson: 0000-0001-8232-8058
Eric M. Bachelder: 0000-0002-8572-888X

\section{Author Contributions}

${ }^{\nabla}$ M.A.C. and R.D.J. contributed equally to this manuscript

\section{Notes}

The authors declare the following competing financial interest(s): Drs. Ainslie, Ting, and Bachelder serve on the advisory board for IMMvention Therapeutix, Inc. Although a financial conflict of interest was identified for management based on the overall scope of the project and its potential benefit to IMMvention Therapeutix, Inc., the research findings included in the publication may not necessarily relate to the interests of IMMvention Therapeutix, Inc. The terms of this arrangement have been reviewed by the University of North Carolina at Chapel Hill in accordance with its policy on objectivity in research.

\section{ACKNOWLEDGMENTS}

This work was supported by funds from the National Institutes of Health U19AI109784 and U54CA198999. The authors would like to acknowledge the assistance from the UNC Chapel Hill Analytical and Nanofabrication Laboratory (CHANL) in acquiring SEM images and would also like to thank the UNC CFAR Virology, Immunology, and Microbiology Core for use of their ELISpot reader. Cell lines (Madin-Darby canine kidney (MDCK) cells, London Line, and FR-58) were obtained through the Influenza Reagent Resource, Influenza Division, WHO Collaborating Center for Surveillance, Epidemiology and Control of Influenza (Centers for Disease Control and Prevention, Atlanta, GA, USA). Endpoint ELISA assays were performed in the Immunology Unit of the Duke Regional Biocontainment Laboratory, which received partial support for construction from the National Institutes of Health, National Institute of Allergy and Infectious Diseases (UC6-AI058607). The authors would also like to acknowledge the valuable technical assistance of: Dr. Willie June Brickey, Kimberly Parks, Kristina Riebe, Melissa Samo, and Christopher Sample.

\section{REFERENCES}

(1) Pliaka, V.; Kyriakopoulou, Z.; Markoulatos, P. Risks associated with the use of live-attenuated vaccine poliovirus strains and the strategies for control and eradication of paralytic poliomyelitis. Expert Rev. Vaccines 2012, 11 (5), 609-28.

(2) Minor, P. D. Live attenuated vaccines: Historical successes and current challenges. Virology 2015, 479-480, 379-92.

(3) Riese, P.; Schulze, K.; Ebensen, T.; Prochnow, B.; Guzman, C. A. Vaccine adjuvants: key tools for innovative vaccine design. Curr. Top. Med. Chem. 2013, 13 (20), 2562-80.

(4) O’Hagan, D. T.; Ott, G. S.; De Gregorio, E.; Seubert, A. The mechanism of action of MF59 - an innately attractive adjuvant formulation. Vaccine 2012, 30 (29), 4341-8.

(5) Awate, S.; Babiuk, L. A.; Mutwiri, G. Mechanisms of action of adjuvants. Front. Immunol. 2013, 4, 114.

(6) Melchjorsen, J. Learning from the messengers: innate sensing of viruses and cytokine regulation of immunity - clues for treatments and vaccines. Viruses 2013, 5 (2), 470-527.

(7) Zhang, X.; Shi, H.; Wu, J.; Zhang, X.; Sun, L.; Chen, C.; Chen, Z. J. Cyclic GMP-AMP containing mixed phosphodiester linkages is an endogenous high-affinity ligand for STING. Mol. Cell 2013, 51 (2), 226-235.

(8) Diner, E. J.; Burdette, D. L.; Wilson, S. C.; Monroe, K. M.; Kellenberger, C. A.; Hyodo, M.; Hayakawa, Y.; Hammond, M. C.; Vance, R. E. The innate immune DNA sensor cGAS produces a 
noncanonical cyclic dinucleotide that activates human STING. Cell Rep. 2013, 3 (5), 1355-61.

(9) Miyabe, H.; Hyodo, M.; Nakamura, T.; Sato, Y.; Hayakawa, Y.; Harashima, H. A new adjuvant delivery system 'cyclic di-GMP/ YSK05 liposome' for cancer immunotherapy. J. Controlled Release 2014, 184, 20-7.

(10) Hanson, M. C.; Crespo, M. P.; Abraham, W.; Moynihan, K. D.; Szeto, G. L.; Chen, S. H.; Melo, M. B.; Mueller, S.; Irvine, D. J. Nanoparticulate STING agonists are potent lymph node-targeted vaccine adjuvants. J. Clin. Invest. 2015, 125 (6), 2532-46.

(11) Nakamura, T.; Miyabe, H.; Hyodo, M.; Sato, Y.; Hayakawa, Y.; Harashima, H. Liposomes loaded with a STING pathway ligand, cyclic di-GMP, enhance cancer immunotherapy against metastatic melanoma. J. Controlled Release 2015, 216, 149-57.

(12) Goodwin, T. J.; Huang, L. Investigation of phosphorylated adjuvants co-encapsulated with a model cancer peptide antigen for the treatment of colorectal cancer and liver metastasis. Vaccine 2017, 35 (19), 2550-2557.

(13) Lee, E.; Jang, H. E.; Kang, Y. Y.; Kim, J.; Ahn, J. H.; Mok, H. Submicron-sized hydrogels incorporating cyclic dinucleotides for selective delivery and elevated cytokine release in macrophages. Acta Biomater. 2016, 29, 271-281.

(14) Wilson, D. R.; Sen, R.; Sunshine, J. C.; Pardoll, D. M.; Green, J. J.; Kim, Y. J. Biodegradable STING agonist nanoparticles for enhanced cancer immunotherapy. Nanomedicine 2018, 14 (2), 237246.

(15) Irvine, D. J.; Hanson, M. C.; Rakhra, K.; Tokatlian, T. Synthetic Nanoparticles for Vaccines and Immunotherapy. Chem. Rev. 2015, 115 (19), 11109-46.

(16) Wang, C.; Ge, Q.; Ting, D.; Nguyen, D.; Shen, H. R.; Chen, J.; Eisen, H. N.; Heller, J.; Langer, R.; Putnam, D. Molecularly engineered poly(ortho ester) microspheres for enhanced delivery of DNA vaccines. Nat. Mater. 2004, 3 (3), 190-6.

(17) Jiang, W.; Gupta, R. K.; Deshpande, M. C.; Schwendeman, S. P. Biodegradable poly(lactic-co-glycolic acid) microparticles for injectable delivery of vaccine antigens. Adv. Drug Delivery Rev. 2005, 57 (3), 391-410.

(18) Bachelder, E. M.; Beaudette, T. T.; Broaders, K. E.; Dashe, J.; Frechet, J. M. Acetal-derivatized dextran: an acid-responsive biodegradable material for therapeutic applications. J. Am. Chem. Soc. 2008, 130 (32), 10494-5.

(19) Broaders, K. E.; Cohen, J. A.; Beaudette, T. T.; Bachelder, E. M.; Frechet, J. M. Acetalated dextran is a chemically and biologically tunable material for particulate immunotherapy. Proc. Natl. Acad. Sci. U. S. A. 2009, 106 (14), 5497-502.

(20) Bachelder, E. M.; Pino, E. N.; Ainslie, K. M. Acetalated Dextran: A Tunable and Acid-Labile Biopolymer with Facile Synthesis and a Range of Applications. Chem. Rev. 2017, 117 (3), 1915-1926.

(21) Kohane, D. S. Microparticles and nanoparticles for drug delivery. Biotechnol. Bioeng. 2007, 96 (2), 203-9.

(22) Junkins, R. D.; Gallovic, M. D.; Johnson, B. M.; Collier, M. A.; Watkins-Schulz, R.; Cheng, N.; David, C. N.; McGee, C. E.; Sempowski, G. D.; Shterev, I.; McKinnon, K.; Bachelder, E. M.; Ainslie, K. M.; Ting, J. P. A robust microparticle platform for a STING-targeted adjuvant that enhances both humoral and cellular immunity during vaccination. J. Controlled Release 2018, 270, 1-13.

(23) Chen, N.; Peine, K.; Collier, M.; Gautam, S.; Jablonski, K.; Guerau-de-Arellano, M.; Ainslie, K.; Bachelder, E. Co-Delivery of Disease Associated Peptide and Rapamycin via Acetalated Dextran Microparticles for Treatment of Multiple Sclerosis. Advanced Biosystems 2017, 1, 1700022.

(24) Chen, N.; Johnson, M. M.; Collier, M. A.; Gallovic, M. D.; Bachelder, E. M.; Ainslie, K. M. Tunable degradation of acetalated dextran microparticles enables controlled vaccine adjuvant and antigen delivery to modulate adaptive immune responses. J. Controlled Release 2018, 273, 147-159.

(25) Bachelder, E. M.; Beaudette, T. T.; Broaders, K. E.; Fréchet, J. M.; Albrecht, M. T.; Mateczun, A. J.; Ainslie, K. M.; Pesce, J. T.; Keane-Myers, A. M. In vitro analysis of acetalated dextran microparticles as a potent delivery platform for vaccine adjuvants. Mol. Pharmaceutics 2010, 7 (3), 826-35.

(26) Peine, K. J.; Bachelder, E. M.; Vangundy, Z.; Papenfuss, T.; Brackman, D. J.; Gallovic, M. D.; Schully, K.; Pesce, J.; Keane-Myers, A.; Ainslie, K. M. Efficient delivery of the toll-like receptor agonists polyinosinic:polycytidylic acid and CpG to macrophages by acetalated dextran microparticles. Mol. Pharmaceutics 2013, 10 (8), 2849-57.

(27) Chen, N.; Collier, M. A.; Gallovic, M. D.; Collins, G. C.; Sanchez, C. C.; Fernandes, E. Q.; Bachelder, E. M.; Ainslie, K. M. Degradation of acetalated dextran can be broadly tuned based on cyclic acetal coverage and molecular weight. Int. J. Pharm. 2016, 512 (1), 147-157.

(28) Duong, A. D.; Sharma, S.; Peine, K. J.; Gupta, G.; Satoskar, A. R.; Bachelder, E. M.; Wyslouzil, B. E.; Ainslie, K. M. Electrospray encapsulation of toll-like receptor agonist resiquimod in polymer microparticles for the treatment of visceral leishmaniasis. Mol. Pharmaceutics 2013, 10 (3), 1045-55.

(29) Schully, K. L.; Bell, M. G.; Prouty, A. M.; Gallovic, M. D.; Gautam, S.; Peine, K. J.; Sharma, S.; Bachelder, E. M.; Pesce, J. T.; Elberson, M. A.; Ainslie, K. M.; Keane-Myers, A. Evaluation of a biodegradable microparticulate polymer as a carrier for Burkholderia pseudomallei subunit vaccines in a mouse model of melioidosis. Int. J. Pharm. 2015, 495 (2), 849-61.

(30) Schully, K. L.; Sharma, S.; Peine, K. J.; Pesce, J.; Elberson, M. A.; Fonseca, M. E.; Prouty, A. M.; Bell, M. G.; Borteh, H.; Gallovic, M.; Bachelder, E. M.; Keane-Myers, A.; Ainslie, K. M. Rapid vaccination using an acetalated dextran microparticulate subunit vaccine confers protection against triplicate challenge by bacillus anthracis. Pharm. Res. 2013, 30 (5), 1349-61.

(31) Kayamuro, H.; Yoshioka, Y.; Abe, Y.; Arita, S.; Katayama, K.; Nomura, T.; Yoshikawa, T.; Kubota-Koketsu, R.; Ikuta, K.; Okamoto, S.; Mori, Y.; Kunisawa, J.; Kiyono, H.; Itoh, N.; Nagano, K.; Kamada, H.; Tsutsumi, Y.; Tsunoda, S. Interleukin-1 family cytokines as mucosal vaccine adjuvants for induction of protective immunity against influenza virus. J. Virol 2010, 84 (24), 12703-12.

(32) Ichinohe, T.; Lee, H. K.; Ogura, Y.; Flavell, R.; Iwasaki, A. Inflammasome recognition of influenza virus is essential for adaptive immune responses. J. Exp. Med. 2009, 206 (1), 79-87.

(33) Arulanandam, B. P.; Mittler, J. N.; Lee, W. T.; O’Toole, M.; Metzger, D. W. Neonatal administration of IL-12 enhances the protective efficacy of antiviral vaccines. J. Immunol. 2000, 164 (7), 3698-704.

(34) Arulanandam, B. P.; O’Toole, M.; Metzger, D. W. Intranasal interleukin-12 is a powerful adjuvant for protective mucosal immunity. J. Infect. Dis. 1999, 180 (4), 940-9.

(35) Tan, R. S.; Ho, B.; Leung, B. P.; Ding, J. L. TLR cross-talk confers specificity to innate immunity. Int. Rev. Immunol. 2014, 33 (6), 443-53.

(36) Querec, T.; Bennouna, S.; Alkan, S.; Laouar, Y.; Gorden, K.; Flavell, R.; Akira, S.; Ahmed, R.; Pulendran, B. Yellow fever vaccine YF-17D activates multiple dendritic cell subsets via TLR2, 7, 8, and 9 to stimulate polyvalent immunity. J. Exp. Med. 2006, 203 (2), 41324.

(37) Kawai, T.; Akira, S. Toll-like receptors and their crosstalk with other innate receptors in infection and immunity. Immunity 2011, 34 (5), 637-50.

(38) Kauffman, K. J.; Do, C.; Sharma, S.; Gallovic, M. D.; Bachelder, E. M.; Ainslie, K. M. Synthesis and characterization of acetalated dextran polymer and microparticles with ethanol as a degradation product. ACS Appl. Mater. Interfaces 2012, 4 (8), 4149-55.

(39) Gallovic, M. D.; Montjoy, D. G.; Collier, M. A.; Do, C.; Wyslouzil, B. E.; Bachelder, E. M.; Ainslie, K. M. Chemically modified inulin microparticles serving dual function as a protein antigen delivery vehicle and immunostimulatory adjuvant. Biomater. Sci. 2016, 4 (3), 483-93.

(40) Brito, L. A.; Singh, M. Acceptable levels of endotoxin in vaccine formulations during preclinical research. J. Pharm. Sci. 2011, 100 (1), $34-7$. 
(41) Lutz, M. B.; Kukutsch, N.; Ogilvie, A. L.; Rossner, S.; Koch, F.; Romani, N.; Schuler, G. An advanced culture method for generating large quantities of highly pure dendritic cells from mouse bone marrow. J. Immunol. Methods 1999, 223 (1), 77-92.

(42) Samo, M.; Choudhary, N. R.; Riebe, K. J.; Shterev, I.; Staats, H. F.; Sempowski, G. D.; Leduc, I. Immunization with the Haemophilus ducreyi trimeric autotransporter adhesin DsrA with alum, CpG or imiquimod generates a persistent humoral immune response that recognizes the bacterial surface. Vaccine 2016, 34 (9), 1193-1200.

(43) Guglani, L.; Khader, S. A. Th17 cytokines in mucosal immunity and inflammation. Curr. Opin. HIV AIDS 2010, 5 (2), 120-7.

(44) Schmitz, N.; Kurrer, M.; Bachmann, M. F.; Kopf, M. Interleukin-1 is responsible for acute lung immunopathology but increases survival of respiratory influenza virus infection. J. Virol 2005, 79 (10), 6441-8.

(45) Huang, T.; Zhao, K.; Zhang, Z.; Tang, C.; Zhang, X.; Yue, B. DNA vaccination based on pyolysin co-immunized with IL-1beta enhances host antibacterial immunity against Trueperella pyogenes infection. Vaccine 2016, 34 (30), 3469-77.

(46) Staats, H. F.; Ennis, F. A., Jr. IL-1 is an effective adjuvant for mucosal and systemic immune responses when coadministered with protein immunogens. J. Immunol 1999, 162 (10), 6141-6147.

(47) Metzger, D. W. Interleukin-12 as an adjuvant for induction of protective antibody responses. Cytokine+ 2010, 52 (1-2), 102-107.

(48) Stevceva, L.; Moniuszko, M.; Grazia Ferrari, M. Utilizing IL-12, IL-15 and IL-7 as mucosal vaccine adjuvants. Letters in drug design \& discovery 2006, 3 (8), 586-592.

(49) Tomai, M. A.; Miller, R. L.; Lipson, K. E.; Kieper, W. C.; Zarraga, I. E.; Vasilakos, J. P. Resiquimod and other immune response modifiers as vaccine adjuvants. Expert Rev. Vaccines 2007, 6 (5), 83547.

(50) Makela, S. M.; Osterlund, P.; Julkunen, I. TLR ligands induce synergistic interferon-beta and interferon-lambdal gene expression in human monocyte-derived dendritic cells. Mol. Immunol. 2011, 48 (4), $505-15$.

(51) Duong, A. D.; Sharma, S.; Peine, K. J.; Gupta, G.; Satoskar, A. R.; Bachelder, E. M.; Wyslouzil, B. E.; Ainslie, K. M. Electrospray encapsulation of toll-like receptor agonist resiquimod in polymer microparticles for the treatment of visceral leishmaniasis. Mol. Pharmaceutics 2013, 10 (3), 1045-1055.

(52) Gallovic, M. D.; Schully, K. L.; Bell, M. G.; Elberson, M. A.; Palmer, J. R.; Darko, C. A.; Bachelder, E. M.; Wyslouzil, B. E.; KeaneMyers, A. M.; Ainslie, K. M. Acetalated Dextran Microparticulate Vaccine Formulated via Coaxial Electrospray Preserves Toxin Neutralization and Enhances Murine Survival Following Inhalational Bacillus Anthracis Exposure. Adv. Healthcare Mater. 2016, 5 (20), 2617-2627.

(53) Temizoz, B.; Kuroda, E.; Ohata, K.; Jounai, N.; Ozasa, K.; Kobiyama, K.; Aoshi, T.; Ishii, K. J. TLR9 and STING agonists synergistically induce innate and adaptive type-II IFN. Eur. J. Immunol. 2015, 45 (4), 1159-69.

(54) Yildiz, S.; Alpdundar, E.; Gungor, B.; Kahraman, T.; Bayyurt, B.; Gursel, I.; Gursel, M. Enhanced immunostimulatory activity of cyclic dinucleotides on mouse cells when complexed with a cellpenetrating peptide or combined with CpG. Eur. J. Immunol. 2015, 45 (4), 1170-9.

(55) Kim, Y.; Lee Chung, B.; Ma, M.; Mulder, W. J.; Fayad, Z. A.; Farokhzad, O. C.; Langer, R. Mass production and size control of lipid-polymer hybrid nanoparticles through controlled microvortices. Nano Lett. 2012, 12 (7), 3587-91.

(56) Peine, K. J.; Guerau-de-Arellano, M.; Lee, P.; Kanthamneni, N.; Severin, M.; Probst, G. D.; Peng, H.; Yang, Y.; Vangundy, Z.; Papenfuss, T. L.; Lovett-Racke, A. E.; Bachelder, E. M.; Ainslie, K. M. Treatment of experimental autoimmune encephalomyelitis by codelivery of disease associated Peptide and dexamethasone in acetalated dextran microparticles. Mol. Pharmaceutics 2014, 11 (3), 828-35.

(57) Kasturi, S. P.; Skountzou, I.; Albrecht, R. A.; Koutsonanos, D.; Hua, T.; Nakaya, H. I.; Ravindran, R.; Stewart, S.; Alam, M.; Kwissa,
M.; Villinger, F.; Murthy, N.; Steel, J.; Jacob, J.; Hogan, R. J.; GarciaSastre, A.; Compans, R.; Pulendran, B. Programming the magnitude and persistence of antibody responses with innate immunity. Nature 2011, 470 (7335), 543-7.

(58) Johansen, P.; Martinez Gomez, J. M.; Gander, B. Development of synthetic biodegradable microparticulate vaccines: a roller coaster story. Expert Rev. Vaccines 2007, 6 (4), 471-4.

(59) Joshi, V. B.; Geary, S. M.; Salem, A. K. Biodegradable particles as vaccine delivery systems: size matters. AAPS J. 2013, 15 (1), 8594.

(60) Wang, Y.; Qu, W.; Choi, S. H. FDA's Regulatory Science Program for Generic PLA/ PLGA-Based Drug Products. American Pharmaceutical Review [Online] 2016.

(61) Foucquier, J.; Guedj, M. Analysis of drug combinations: current methodological landscape. Pharmacol. Res. Perspect. 2015, 3 (3), e00149.

(62) Vidarsson, G.; Dekkers, G.; Rispens, T. IgG subclasses and allotypes: from structure to effector functions. Front. Immunol. 2014, $5,520$.

(63) Shibaki, A.; Katz, S. I. Induction of skewed Th1/Th2 T-cell differentiation via subcutaneous immunization with Freund's adjuvant. Exp. Dermatol. 2002, 11 (2), 126-34.

(64) Savelkoul, H. F.; Ferro, V. A.; Strioga, M. M.; Schijns, V. E. Choice and Design of Adjuvants for Parenteral and Mucosal Vaccines. Vaccines 2015, 3 (1), 148-71.

(65) Tel, J.; Lambeck, A. J.; Cruz, L. J.; Tacken, P. J.; de Vries, I. J.; Figdor, C. G. Human plasmacytoid dendritic cells phagocytose, process, and present exogenous particulate antigen. J. Immunol. 2010, $184(8), 4276-83$.

(66) Tacken, P. J.; Zeelenberg, I. S.; Cruz, L. J.; van Hout-Kuijer, M. A.; van de Glind, G.; Fokkink, R. G.; Lambeck, A. J.; Figdor, C. G. Targeted delivery of TLR ligands to human and mouse dendritic cells strongly enhances adjuvanticity. Blood 2011, 118 (26), 6836-44.

(67) Cruz, L. J.; Tacken, P. J.; Pots, J. M.; Torensma, R.; Buschow, S. I.; Figdor, C. G. Comparison of antibodies and carbohydrates to target vaccines to human dendritic cells via DC-SIGN. Biomaterials 2012, 33 (16), 4229-39.

(68) Zhu, Q.; Talton, J.; Zhang, G.; Cunningham, T.; Wang, Z.; Waters, R. C.; Kirk, J.; Eppler, B.; Klinman, D. M.; Sui, Y.; Gagnon, S.; Belyakov, I. M.; Mumper, R. J.; Berzofsky, J. A. Large intestinetargeted, nanoparticle-releasing oral vaccine to control genitorectal viral infection. Nat. Med. 2012, 18 (8), 1291-6.

(69) Silva, J. M.; Zupancic, E.; Vandermeulen, G.; Oliveira, V. G.; Salgado, A.; Videira, M.; Gaspar, M.; Graca, L.; Préat, V.; Florindo, H. F. In vivo delivery of peptides and Toll-like receptor ligands by mannose-functionalized polymeric nanoparticles induces prophylactic and therapeutic anti-tumor immune responses in a melanoma model. J. Controlled Release 2015, 198, 91-103.

(70) Siefert, A. L.; Caplan, M. J.; Fahmy, T. M. Artificial bacterial biomimetic nanoparticles synergize pathogen-associated molecular patterns for vaccine efficacy. Biomaterials 2016, 97, 85-96.

(71) Madan-Lala, R.; Pradhan, P.; Roy, K. Combinatorial Delivery of Dual and Triple TLR Agonists via Polymeric Pathogen-like Particles Synergistically Enhances Innate and Adaptive Immune Responses. Sci. Rep. 2017, 7 (1), 2530. 